University of Nebraska - Lincoln

DigitalCommons@University of Nebraska - Lincoln

January 2007

\title{
Review of Ferroelectric Domain Imaging by Piezoresponse Force Microscopy
}

Andrei Kholkin

University of Aveiro (Portugal), kholkin@cv.ua.pt

Sergei Kalinin

Oak Ridge National Laboratory, sergei2@ornl.gov

Andreas Roelofs

Seagate Technology (Pittsburgh, PA), aroelofs@anl.gov

Alexei Gruverman

University of Nebraska-Lincoln, agruverman2@unl.edu

Follow this and additional works at: https://digitalcommons.unl.edu/physicsgruverman

Part of the Physics Commons

Kholkin, Andrei; Kalinin, Sergei; Roelofs, Andreas; and Gruverman, Alexei, "Review of Ferroelectric Domain Imaging by Piezoresponse Force Microscopy" (2007). Alexei Gruverman Publications. 46.

https://digitalcommons.unl.edu/physicsgruverman/46

This Article is brought to you for free and open access by the Research Papers in Physics and Astronomy at DigitalCommons@University of Nebraska - Lincoln. It has been accepted for inclusion in Alexei Gruverman Publications by an authorized administrator of DigitalCommons@University of Nebraska - Lincoln. 
Published in:

Scanning Probe Microscopy: Electrical and Electromechanical Phenomena at the Nanoscale, Sergei Kalinin and Alexei Gruverman, editors, 2 volumes (New York: Springer Science+Business Media, 2007).

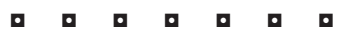

Andrei Kholkin, University of Aveiro (Portugal)

Sergei Kalinin, Oak Ridge National Laboratory

Andreas Roelofs, Seagate Technology (Pittsburgh, PA)

Alexei Gruverman, University of Nebraska-Lincoln

This document is not subject to copyright. 


\title{
I.6
}

\section{Review of Ferroelectric Domain Imaging by Piezoresponse Force Microscopy}

\author{
A. L. Kholkin, S. V. Kalinin, A. RoElofs, AND A. GRUVERMAN
}

This chapter describes the principles, theoretical background, recent developments, and applications of a local probe-based technique for nondestructive highresolution ferroelectric domain imaging and manipulation-piezoresponse force microscopy (PFM). This technique has proven to be a powerful tool for the characterization of ferroelectric thin films, ceramics, and single crystals. Recent advances in application of PFM for studying a mechanism of polarization reversal at the nanoscale, domain dynamics, degradation effects, and size-dependent phenomena in ferroelectrics are reviewed in detail. Examples of using PFM for the characterization of various polar materials such as ferroelectric films, piezoelectric semiconductors, and ferroelectric relaxors are given.

\section{Introduction}

Ferroelectric materials attract significant attention because they have a number of applications in electronic devices. One of the main features of ferroelectrics is the presence of two or more thermodynamically equivalent switchable polarization states, which can be employed in nonvolatile random access memory (FeRAM) devices and for high-density data storage. FeRAM is one of the most promising and advanced nonvolatile memory technologies in use today. It offers such benefits as high reading and writing speed, low power consumption, high endurance, and better scalability than other nonvolatile memories [1]. For FeRAM to become high-volume, mainstream devices competitive with other types of nonvolatile memories, two major goals must be accomplished. First is the ability to manufacture at a high level of device integration (i.e., memory density in the gigabit range at least), where the constituent ferroelectric capacitors should be reduced to submicron dimensions. Second, it is necessary to ensure that the FeRAM devices of these dimensions can achieve the necessary performance, reliability, and lifetime requirements. This necessitates that several fundamental issues be addressed, including the effects of film thickness and lateral size of the ferroelectric capacitor on ferroelectric properties such as remanent polarization and coercive field. Also, of fundamental interest is the relationship between the capacitor size and the 
mechanism of degradation effects such as retention, imprint and polarization loss during operation (fatigue).

To answer these questions, one has to probe ferroelectric domain configurations and local properties at micro- and nanometer scales, since the investigation of domain structure and its evolution during polarization reversal provides full and direct information on the static and dynamic properties of ferroelectrics. Scanning probe microscopy (SPM) offers several different possibilities to reveal information on the domain configurations of ferroelectric thin films and single crystals. Several reviews of SPM-based methods for characterization of ferroelectric domains are available in the literature [2,3]. A difference in mechanical, structural, electrochemical, dielectric, and piezoelectric properties of opposite ferroelectric domains may provide distinct domain contrast in the SPM contact mode. In the noncontact regime, domains can be visualized by detecting surface polarization charge and surface potential.

Currently, the most widely used SPM method for imaging domain structure of ferroelectric surfaces is piezoresponse force microscopy (PFM) [4-10], based on monitoring piezoelectric surface displacements induced by the electrically biased probing tip. This method was introduced in 1992 by Guethner and Dransfeld [11] to detect polarized regions in ferroelectric copolymer films and, soon after that, proved to be the most effective approach for the nanoscale study and control of ferroelectric domains in bulk crystals and thin films [12,13]. Figure 1 illustrates PFM's

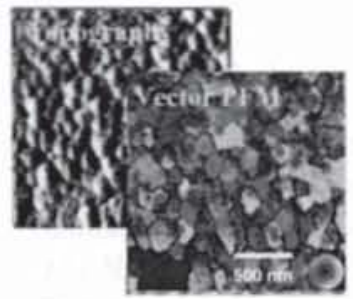

Domain imaging

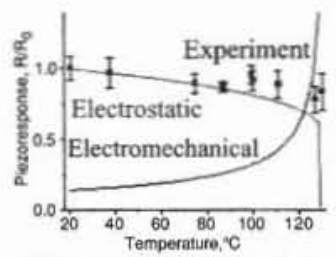

Temperature dynamics and phase transitions

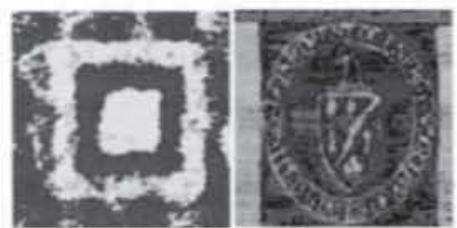

Domain Writing

Piezoresponse Force

Microscopy

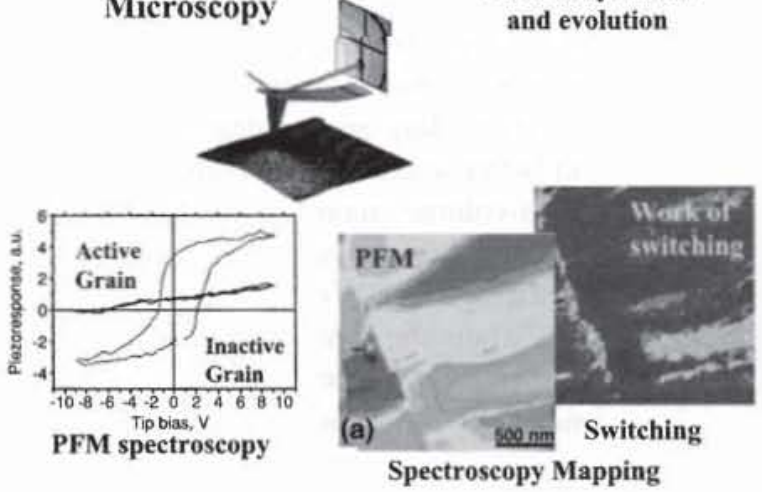

FIGURE 1. Schematic illustration of PFM capabilities in nanoscale characterization of ferroelectrics. (See also Plate 2 in the Color Plate Section.) 


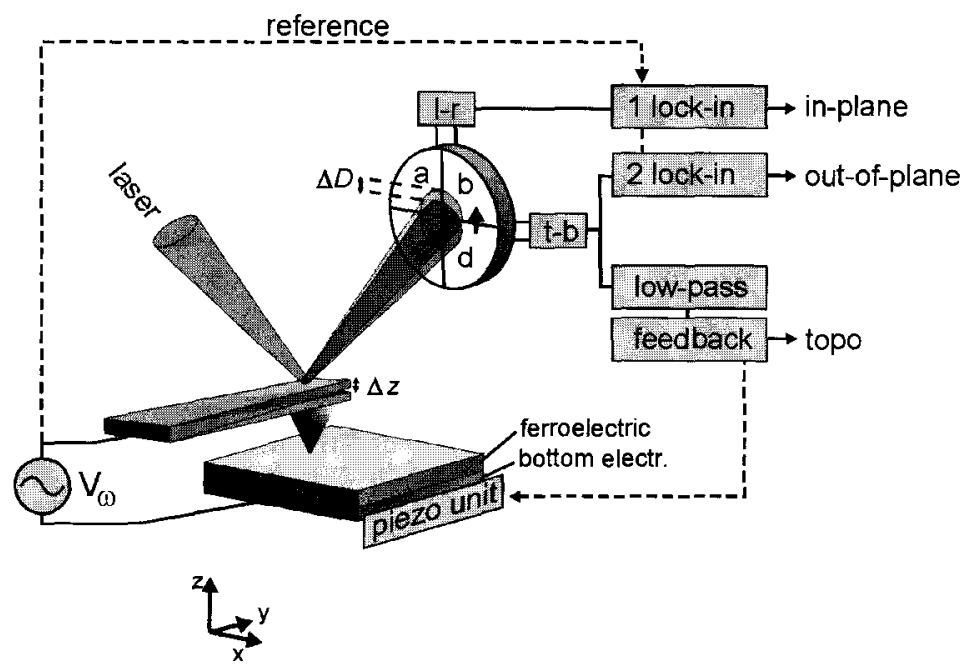

FIGURE 2. PFM experimental setup for simultaneous acquisition of topography and vertical and lateral polarization components. A function generator is used to apply an ac voltage $V_{\omega}$ between the tip and the bottom electrode. The voltage-induced cantilever deflection is detected by a reflected laser beam on a four quadrant photodiode. The two signals: $(a+c)-(b+d)$ and $(a+b)-(c+d)$ are demodulated with two lock-in's representing the in-and out-of-plane signals, respectively. The average force on the cantilever during the scanning process is kept constant by a feedback loop.

capabilities for obtaining information on the nanoscale properties of static domain configurations, domain switching behavior, slow relaxation processes, and local hysteresis spectroscopy. In this chapter, we summarize the basic principles of PFM, show what kind of information can be obtained from PFM experiments, delineate the limitations of PFM signal interpretation relevant to quantitative imaging, and review recent advances in PFM with respect to nanoscale ferroelectric research and applications.

\section{Principle of PFM}

\subsection{Experimental Setup}

The standard experimental PFM setup is usually based on a commercial scanning probe microscope equipped with a four-quadrant photo detector, a conductive probing tip, a function generator, and two lock-in amplifiers (as illustrated in Figure 2).

A ferroelectric sample under investigation is placed between the bottom electrode and the conductive PFM tip, serving as a movable top electrode. The most frequently used probes are either metal-coated silicon probes with conducting 
coatings (Pt/Ir, $\left.\mathrm{WC}_{2}, \mathrm{Au}, \mathrm{Rh}\right)$ or highly doped silicon cantilevers. Measurements are carried out in the contact mode and allow information on the out-of-plane component of electromechanical surface response-i.e., normal to the film plane (vertical PFM, or VPFM), as well as on the in-plane component via the frictional forces (lateral PFM, or LPFM) [14], to be obtained. Application of an ac electric bias to the probing tip results in a sample surface oscillation due to the converse piezoelectric effect. Electronic feedback (that maintains the "average" force on the cantilever constant) facilitates accurate tracking of the surface motion by using the lock-in technique with simultaneous recording of the surface topography. The effect of the Maxwell stress (capacitive forces) compared to the piezoelectric response (electromechanical forces) is discussed in the following section. Typical operation frequencies for VPFM vary from $1-3 \mathrm{kHz}$ to $2-5 \mathrm{MHz}$, as limited by the bandwidth of optical detector. In LPFM, the transduction of signal from surface to the tip necessitates operation in the $1-30 \mathrm{kHz}$ range.

Note that, in order to prevent attenuation of the out-of-plane piezoelectric response signal, the feedback loop has to operate at a frequency lower than the frequency $\omega$ of the applied voltage $V_{\omega}$. This provides the possibility to separate the PFM signal from the topographical information. To avoid the cross-talk between piezoelectric deformation and topographic image, a low pass filter is typically put in front of the feedback. At the same time, high operating frequency ensures sufficient sampling of each image point.

In terms of exciting the piezoelectric vibration of the sample, there are two major approaches in PFM. In a local excitation approach, the vibration is generated locally by applying a modulation voltage between the bottom electrode and the conductive SPM tip which scans the bare surface of the film without a deposited top electrode (Figure 3(a)). An advantage of this approach is the possibility of establishing correlations between domain configurations and film microstructure. In addition, this method can be used for nanoscale domain control via highly localized polarization reversal and direct investigation of domain wall interaction with microstructural features, such as defects and grain boundaries, for local spectroscopy measurements, and for the investigation of electrical and mechanical coupling between adjacent grains. Furthermore, this approach offers extremely high resolution, potentially allowing for the investigation of the microscopic mechanism of the domain wall motion.

Note that the electric field generated by the SPM tip in this configuration is highly inhomogeneous, which makes quantitative analysis of the field- induced signal extremely difficult. In other words, PFM measurements in the sample without the extended top electrode collect signal only from a surface layer of an unknown thickness that is a function of dielectric permittivity and contact conditions. ${ }^{1}$ This effect has been understood by directly comparing PFM images taken on epitaxial PZT thin films containing $a$-domains with a cross-sectional TEM image

\footnotetext{
${ }^{1}$ Only when the diameter of the tip-sample contact area is of the same order of magnitude as the film thickness can the field be treated as approximately homogeneous. This is valid for standard cantilevers and films with the thicknesses below $\sim 20 \mathrm{~nm}$.
} 


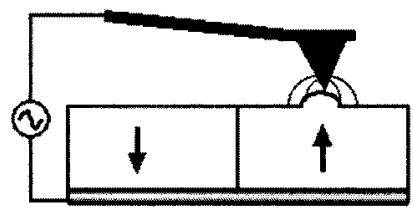

(a)

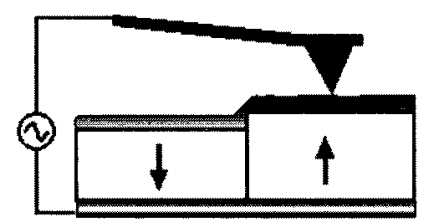

(b)

FIGURE 3. Local (a) and integral (b) methods of excitation in PFM.

[15]. It was found that the $a$-domain width observed in the PFM measurement complies with the $a$-domain width near the surface determined from the TEM image. This proves that the PFM measurement is not integrating over the whole film thickness but images the polarization only several nanometers below the surface.

In a global excitation approach, domain structure can be visualized through the top electrode of a ferroelectric capacitor (although at the expense of lower lateral resolution). In this case, the piezoelectric vibration is generated in a film region underneath the deposited top electrode which is much larger that the tip-sample contact area. The modulation voltage can be applied either by using an external wire attached to the top electrode or, in the case of a micrometer size electrode, directly through the conductive SPM tip. As in the former case, the piezoelectric displacement is probed locally by the SPM tip (Figure 3(b)). In such a configuration, a homogeneous electric field is generated throughout the ferroelectric film, which allows quantitative treatment of domain wall dynamics and investigation of polarization reversal mechanism in ferroelectric capacitors. Due to the reduced time constant, fast pulse switching and transient current measurements can be accomplished in submicron capacitors, thus making PFM suitable for ferroelectric memory device testing. Hereafter, to distinguish between samples without and with top electrodes we will refer to the latter as "capacitors."

\subsection{Elementary Theory of PFM}

Even though the PFM technique was introduced more than ten years ago, the quantitative analysis of PFM measurements is still under debate [16-20]. The three major issues involved in PFM contrast formation mechanism are (i) voltagedependent contact mechanics of piezoelectric material that determines the PFM signal, (ii) dynamic properties of the cantilever and transduction mechanism between the electromechanical response of the surface and electrostatic forces and resulting vertical and lateral PFM signal, and (iii) electroelastic field distribution in the material that determines resolution, domain switching behavior, and hysteresis loop acquisition mechanism.

Luo et al. [21] have found that the piezoelectric (1st harmonics) signal of ferroelectric triglycine sulfate does not diverge at the phase transition but gradually 
decreases, following the same trend as the spontaneous polarization. From this fact the authors concluded that the signal was dominated by the capacitive forces, rather than by electromechanical ones, since the electromechanical signal was expected to be proportional to the relevant piezoelectric coefficient that should strongly increase in the vicinity of the phase transition. The influence of the capacitive force was further supported by the finite vibration amplitude measured on non-ferroelectric surfaces [22]. On the other hand, the ability to detect the in-plane piezoelectric signal, the absence of PFM signal attenuation due to charge screening, and the possibility of domain imaging in ferroelectric capacitors through the top electrode are all indicative of a strong contribution of the electromechanical forces to the observed PFM contrast.

As such, taking both the electromechanical response and the long-range capacitive forces into account, we can present the total response of a cantilever in a contact with ferroelectric in a form: $A=A_{\text {piezo }}+A_{c a p}+A_{n l}$, where $A_{\text {piezo }}$ represents the electromechanical response of the surface due to converse piezoelectric effect, $A_{\text {cap }}$ is the contribution due to the electrostatic force between tip and sample, and $A_{n l}$ is the contribution due to non-local electrostatic force between the cantilever and the sample. Typically, when the domain size is much smaller than the cantilever length, non-local interaction results only in a constant background (offset) that can be easily subtracted from PFM image data.

In order to get reliable piezoelectric response data, one has to maximize the electromechanical contribution and to minimize the electrostatic one. One of the most serious problems affecting the electromechanical signal is an interfacial layer (dielectric gap) between the PFM tip and the ferroelectric surface [23] due to either sample surface contamination or oxidation of the Si probe. To get a good electrical contact between the tip and the sample surface one has to clean the surface of the ferroelectric by thermal treatment prior the scanning process (heating in vacuum at $200^{\circ} \mathrm{C}$ removes water, $\mathrm{CO}$, and $\mathrm{CO}_{2}$ [24]) and to use metal-coated instead of doped silicon cantilevers. Tip-sample contact force is also an important factor that determines the efficiency of PFM imaging. To overcome the contact problem, Kalinin and Bonnell [8] suggested to use stiff cantilevers (spring constant $k>1$ $\mathrm{N} / \mathrm{m}$ ) and apply high contact forces (on the order of 10 to $1,000 \mathrm{nN}$ depending on the tip radius and the thickness of the dielectric gap). However, this should be used with caution since at high contact forces the direct piezoelectric effect may counteract with the converse piezoelectric effect and additional effects such as higher-order ferroic switching [25] or stress-induced suppression of piezoelectricity [26,27] related to the phase transition under the high mechanical stress exerted by the tip [28]. This makes the analysis of the PFM measurements more complex. In the following, we will briefly analyze the forces acting on the tip while applying a voltage.

\subsubsection{Capacitive forces}

The system comprising the conducting tip in a contact with the dielectric surface represents a capacitor. Therefore, an external voltage applied between the tip and 
the counter electrode results in an additional (capacitive or Maxwell) force that can be, in general, determined by the following equation:

$$
F_{c a p}=\frac{d W_{c a p}}{d z}=\frac{1}{2} V^{2} \frac{d C}{d z},
$$

where $W_{\text {cap }}=V^{2} C / 2$ is the energy stored in the capacitor $C$ and $z$ is the vertical distance. It follows that, if a combination of the dc and ac voltages $\left(V=V_{\mathrm{dc}}+\right.$ $\left.V_{\omega} \cos \omega t\right)$ is applied between the tip and the counter-electrode, the capacitive forces will consist of a static force $F_{0}$, a force oscillating at a driving frequency $\omega\left(F_{\omega}=V_{d c} V_{\omega} d C / d z\right)$ and a force $F_{2 \omega}$ appearing at a frequency $2 \omega$. These forces can be essential even in contact mode [18] and must be compared with other forces, including the electromechanical one due to electric field-induced deformation of the sample.

The total capacitance $C$ of the sample-cantilever system can be written as the sum of the capacitance of the cantilever $\left(\mathrm{C}_{\text {cant }}\right)$ and the capacitance of the tip $\left(C_{t i p}\right)$. The former gives rise to so-called nonlocal contribution to the capacitive force because of the macroscopic dimensions of the cantilever. The capacitance of the cantilever can be estimated by using the analytical expression derived by Prume et al. [29]:

$$
\frac{d C_{c a n t}}{d z}=\frac{\varepsilon_{0} d_{c}}{\tan \alpha}\left(\frac{1}{h_{c}}-\frac{1}{L_{c} \tan \alpha+h_{c}}\right),
$$

where $d_{c}$ is the width and $L_{c}$ is the length of the cantilever. $h_{c}$ and $\alpha$ are the gap and the angle between the cantilever and the sample surface, respectively. Equation 2 describes the non-local electrostatic force contribution $F_{\mathrm{nl}}$ of the cantilever to the PFM signal and can result in a signal offset appearing at 1 st harmonics (frequency $\omega)^{2}$. The typical value of the dc force for nonlocal capacitive contribution (standard Si cantilevers, $L_{c}=450 \mu \mathrm{m}, d_{c}=50 \mu \mathrm{m}$ ) estimated using Eq. (2) is quite small $(\sim 0.1 \mathrm{nN})$ and can be neglected for many practical cases.

In addition to capacitive forces, Coulomb attractive forces between the tip and the charged ferroelectric surface can be observed in PFM experiments (Hong et al. [30]). This force can be estimated by using an equation:

$$
F_{\text {coul }}=\frac{\sigma C V}{2 \varepsilon_{0}},
$$

where $\sigma$ is the surface-bound charge density and $V$ is the voltage applied to the sample. Surface polarization charges giving rise to electrostatic interaction are screened by the absorbed charges of the opposite sign and thus are normally smaller than the electromechanical forces for many practical applications. For the typical polarization charge of $1 \mu \mathrm{C} / \mathrm{cm}^{2}$, tip radius $\approx 40 \mathrm{~nm}$, and bias voltage of $V \approx 1 \mathrm{~V}$, the corresponding force can be estimated in the range of few nN.

\footnotetext{
${ }^{2}$ Note that the PFM signal is monitored at a driving frequency $\omega$.
} 
We should finally note that the major attractive forces between the tip and the sample surface are adhesion forces due to Van der Waals interaction. Rabe et al. [31] reported values of $50-500 \mathrm{nN}$ for different types of cantilevers in contact with dielectric and metallic surfaces. This proved that the dominant forces in the PFM measurements should be both adhesive forces and strong short-range repulsive forces $F_{\text {rep }}$. These forces compel the cantilever to follow the motion of the ferroelectric surface caused by the converse piezoelectric effect with Coulomb interaction playing only a minor role.

\subsubsection{Electromechanical Forces}

Applying an electric field between a cantilever and a dielectric material leads to capacitive forces as discussed above. A piezoelectric material will provide an additional response to an applied ac electric field due to the converse piezoelectric and electrostrictive effects. For a homogeneously polarized (in the $z$-direction), stress-free ferroelectric material, its vertical $(Z)$ displacement can be expressed as follows:

$$
\Delta z=d_{33} V+\frac{M_{333}}{t} V^{2}
$$

where $V$ is the applied voltage, $t$ is the sample thickness, and $d_{33}$ and $M_{333}$ are the piezoelectric and electrostrictive constants, respectively. The second term in Eq. (4) is typically much smaller than the first one in a polarized state. [32] Under the external voltage $V=V_{\mathrm{dc}}+V_{\omega} \cos (\omega t)$ the surface displacement will consist of a dc component (that cannot be measured with a lock-in), and the signals at first and second harmonics:

$$
\begin{gathered}
\Delta z_{\omega}=d_{33} V_{\omega}+2 \frac{M_{333}}{t} V_{d c} V_{\omega}, \\
\Delta z_{2 \omega}=\frac{1}{2} V_{\omega}^{2} \frac{M_{333}}{t} .
\end{gathered}
$$

The sign of the converse piezoelectric signal (first term in Eq. (5)) depends on the relative orientation of the polarization and applied electric field. When a positive voltage is applied to the PFM tip and the polarization is pointing at negative $z$ direction (electric field is parallel to the spontaneous polarization), the sample will expand, thus pushing up the cantilever and allowing measurements of $d_{33}$ (Figure $4(a))$. In this case, a positive sign is attributed to the displacement $\Delta z$. As we apply an alternating voltage, this means that the electric field and the piezoelectric signal are in phase. These measurements are referred to as out-of-plane (or vertical PFM) measurements. If the polarization points at the positive $z$-direction, the applied electric field will be antiparallel to the spontaneous polarization, causing the ferroelectric to contract with the consequent lowering of the cantilever (Figure 4(b)). The electric field and the piezoresponse signal are shifted in phase by $180^{\circ}$. Additionally, ferroelectric grain may contract in the film plane, which is caused 

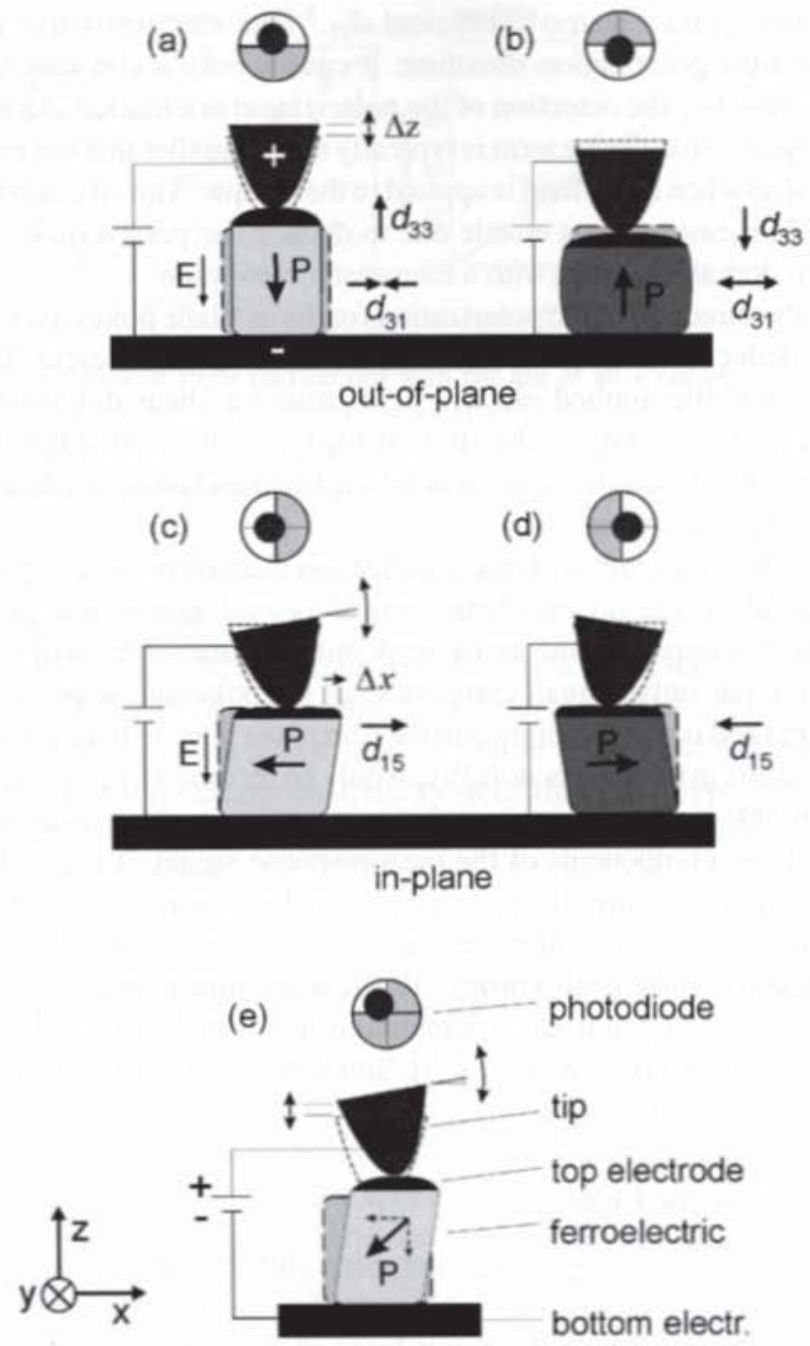

FIGURE 4. Piezoelectric effect in ferroelectric perovskite investigated by PFM. (a) Electric field aligned parallel to the spontaneous polarization leads to a lifting of the cantilever due to the $d_{33}$ effect (out-of-plane signal). It causes additional lateral contraction of the ferroelectric via the $d_{31}$ piezoelectric coefficient. (b) The antiparallel alignment of the electric field and the spontaneous polarization leads to a vertical contraction and a horizontal expansion of the ferroelectric. (c, d) Electric field applied orthogonal to the polarization results in a shear movement due to the $d_{15}$ coefficient. This movement causes a torsional deformation of the cantilever forcing the laser spot to move horizontally (in-plane signal). (e) A grain polarized in the $x$-z-plane will contribute to the in-plane as well as to the out-of-plane signal. 
by the transverse piezoelectric coefficient $d_{31} \cdot{ }^{3}$ The electrostrictive effect does not depend on the polarization direction: it causes only a constant background but does not influence the detection of the polarization orientation. As was already mentioned, the electrostrictive term is typically much smaller that the piezoelectric one and vanishes when no dc field is applied to the sample. Thus it can be concluded that the VPFM measurements enable one to deduce the polarization direction of the individual domain or grain with a nanometer resolution.

Similarly, the direction of the polarization for the in-plane polarized ferroelectric grain can be deduced via a relevant (shear) piezoelectric coefficient (Figure 4(c), (d)). In this case, the applied electric field causes a shear deformation of the grain, which is transferred via the friction forces to the torsional movement of the cantilever. These measurements will be further denoted as in-plane (or lateral PFM, or LPFM) measurements.

Figure 4(e) shows a grain with the polarization aligned in the $x$-z-plane. In this case, the applied field will cause both vertical as well as torsional movement of the cantilever. A strong in-plane and a weak out-of-plane signal will indicate that the polarization has only a small component of the polarization pointing into the $z$-direction $\left(P_{\mathrm{z}}\right)$ and is mainly aligned in the film plane. Due to the cantilever asymmetry, polarization in the $y$-direction $P_{\mathrm{y}}$ can only be recorded by physically rotating the sample by $90^{\circ}$ along the $z$-axis and repeating the in-plane measurement. By acquiring all three components of the piezoresponse signal, it is possible to perform at least semiquantitative reconstruction of polarization orientation. However, precise orientation of polarization can be calculated only if all the components of the piezoelectric tensor are known. The first attempt to relate the amplitude of the piezoresponse signal to the orientation of the ferroelectric polarization has been undertaken by Harnagea et al. [33], and detailed formalism has been later developed by Kalinin et al. [34].

\subsubsection{Calibration of the PFM}

The PFM can be calibrated by using a commercially available $x$-cut quartz. The textbook value of its piezoelectric coefficient $d_{11}=-2.3 \mathrm{pm} / \mathrm{V}$ means that the application of a bias of $1 \mathrm{~V}$ to the major faces of an $x$-cut quarts leads to a contraction of $2.3 \mathrm{pm}$ along the $x$-direction. To measure the converse piezoelectric effect, gold electrodes are deposited onto both sides of the crystal. Afterwards, the

\footnotetext{
${ }^{3}$ This effect could lead to a torsional movement of the cantilever in the case when extended top electrodes are used and the cantilever is not positioned in the center of the grain. This would make the analysis of the three-dimensional polarization distribution more complicated or even impossible, whereas using the tip as a movable top electrode will only cause a symmetrical contraction of the ferroelectric around the tip and consequently causing no torsional movement. The in-plane and out-of-plane measurements will thus exhibit almost no crosstalk.

${ }^{4}$ When a plane parallel plate is cut from a single crystal, the term $c u t$ is used to indicate the direction of the normal to the major faces. Thus in the case of $x$-cut the normal to its major faces is parallel to the $x$-axis of the crystal.
} 
(a) laser interferrometer

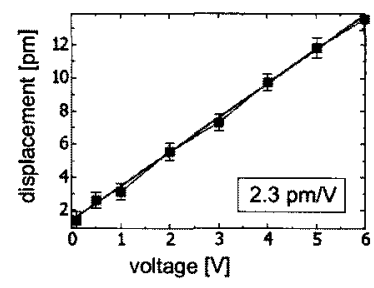

(b) PFM

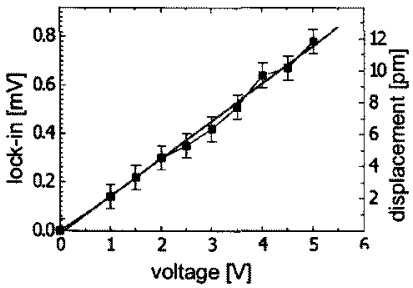

FIGURE 5. Calibration of PFM carried out with the aid of an $x$-cut quartz plate. (a) Measurement of the quartz plate in a double-beam laser interferometer to check the value of the piezoelectric coefficient $d_{11}$. (b) Measurement of the lock-in output in PFM. The PFM signal is interrelated to the known dilation of the quartz sample.

quality of the crystal is checked by performing converse piezoelectric measurements by using a sensitive displacement meter, for example, a double-beam laser interferometer (Figure 5(a)). Then the crystal is placed on the AFM stage and the lock-in output of the PFM is measured as a function of the applied voltage (at a fixed frequency) calibrating it to a known dilation of the quartz (Figure 5(b)). For accurate quantitative measurements, the PFM calibration is repeated for each new mounted cantilever, as the lock-in output is sensitive to the laser alignment and the exact cantilever parameters.

\section{Nanoscale Domain Switching and PFM Spectroscopy}

PFM provides a unique opportunity for direct studies of the domain structure evolution under an external electric field, which cannot be matched by previously available techniques. A conductive probing tip can be used not only for domain visualization but also for modification of the initial domain structure. Application of a small dc voltage between the tip and bottom electrode generates an electric field of several hundred kilovolts per centimeter, which is higher than the coercive voltage of most ferroelectrics, thus inducing local polarization reversal. An application of the positive or negative dc bias to the tip can induce $180^{\circ}$ polarization switching, orienting polarization upward or downward. Thus, created domains can be imaged using PFM, which, therefore, provides both "storage" and "read-out" capabilities. Given that the width of domain walls in ferroelectrics is typically very small (on the order of 1-3 unit cells), this ferroelectric recording [35] potentially allows extremely high data storage density [36,37], well above that achievable by conventional magnetic recording. In fact, 40 -nm bit size had been demonstrated as early as 2002 by Tybell et al. [36], while a similar level was achieved using thermomagnetic recording by Wickramasinghe group only in 2004 [38]. By the end of 2005, Cho et al. [39] had demonstrated 8-nm bit size corresponding to 10 $\mathrm{TBit} / \mathrm{inch}^{2}$ storage density. These considerations have resulted in significant interest to ferroelectric data storage from major industrial corporations, and the review of the current state of the field is provided by Hong and Park in Chapter IV.6. 
Other important applications, such as domain engineering for electrooptical devices, have also emerged [40]. A further extension of this approach is based on the fact that surface chemical reactivity of ferroelectric materials in, e.g., acid dissolution processes is strongly polarization-dependent [41]. Recently, similar behavior was observed for metal photodeposition [42]. The combination of polarizationdependent chemical reactivity and domain patterning gives rise to ferroelectric lithography-a novel approach for nanoscale structure fabrication [43-45]. See also Chapter IV.4 by Bonnell, Lei, and $\mathrm{Li}$ in this book.

\subsection{Thermodynamics of Domain Switching}

Applications of PFM for high-density data storage and ferroelectric lithography necessitate studies of both thermodynamics and kinetics of the switching process. The first analysis of the thermodynamics of domain switching in PFM capturing the essential features of this process including finite nucleation bias was given by Abplanalp [20]. The closed-form solution (Molotskii model) for domain switching in the point charge approximation was given by Molotskii et al. [46,47]. The thermodynamics of domain switching using rigorously derived [48] electroelastic fields was given by Kalinin et al. [49]. Subsequently, the analysis of this problem was further elaborated by Morozovska and Eliseev [50] and by Emelyanov [51]. In all cases, it was realized that the spatial extent of the field produced by the tip is finite; therefore, the switching will be limited to a small volume of material. For point-charge type models with charge located on or below the ferroelectric surface, the field in the vicinity of the tip is infinite and domain nucleation is induced at arbitrarily small biases [47,52]. In the models including finite tip-surface separation or based on realistic field geometries [47,49-51,53], the finite nucleation bias is predicted (Figure 6). Below, we analyze the thermodynamics of domain switching in a more detail.

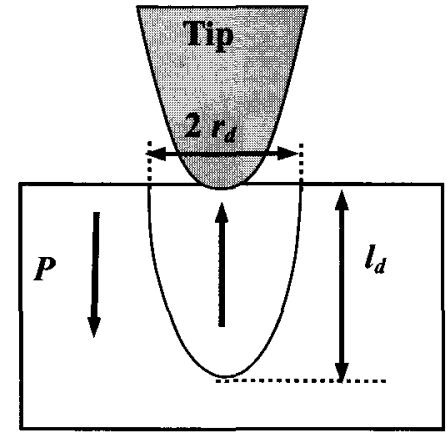

(a)

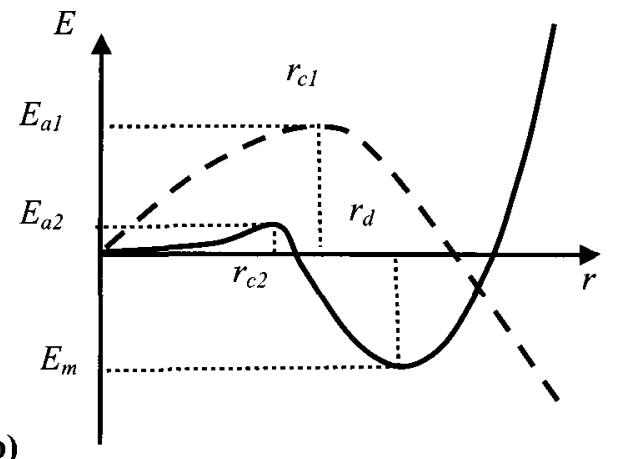

(b)

FIGURE 6. (a) Domain geometry during tip-induced switching. (b) Free energy as a function of the lateral domain size. Dashed line, in a uniform electric field; solid line, in a tip-induced electric field. 
The driving force for the $180^{\circ}$ polarization switching process in ferroelectrics is due to change in the bulk free energy density $[20,25]$ :

$$
\Delta g_{b u l k}=-\Delta P_{i} E_{i}-\Delta d_{i \mu} E_{i} X_{\mu}
$$

where $P_{i}, E_{i}, X_{\mu}$, and $d_{i \mu}$, are the components of the polarization, electric field, stress and piezoelectric constants tensor, respectively, $i=1,2,3$, and $\mu=1, \ldots, 6$. The first and second terms in Eq. (7) describe ferroelectric and ferroelectroelastic switching, respectively. For materials such as $\mathrm{LiNbO}_{3}$ and lead zirconate-titanate (PZT), the signs of the corresponding free energy terms are opposite and the polarities of the domains formed by ferroelectric and ferroelectroelastic switching are opposite, thus providing an approach to distinguish these switching mechanisms.

The free energy of the nucleating domain is

$$
\Delta G=\Delta G_{b u l k}+\Delta G_{w a l l}+\Delta G_{d e p}
$$

where the first term is the change in bulk free energy, $\Delta G_{b u l k}=\int \Delta g_{b u l k} d V$, the second term is the domain wall energy, and the third term is the depolarization field energy. In the Landauer model of switching, the domain shape is approximated as a half-ellipsoid with the small and large axes equal to $r_{d}$ and $l_{d}$, correspondingly (Figure 6(a)). The domain wall contribution to the free energy in this geometry is $\Delta G_{\text {wall }}=b r_{d} l_{d}$, where $b=\sigma_{\text {wall }} \pi^{2} / 2$ and $\sigma_{\text {wall }}$ is the direction-independent domain wall energy. The depolarization energy contribution is $\Delta G_{d e p}=\mathrm{cr}_{d}^{4} / l_{d}$, where

$$
c=\frac{4 \pi P_{s}^{2}}{3 \varepsilon_{11}}\left[\ln \left(\frac{2 l_{d}}{r_{d}} \sqrt{\frac{\varepsilon_{11}}{\varepsilon_{33}}}\right)-1\right]
$$

has only a weak dependence on the domain geometry [54].

In the uniform field case, the free energy surface (as a function of $l_{d}, r_{d}$ ) has a saddle point character and the domain grows indefinitely once the critical size corresponding to activation barrier for nucleation $E_{a}$ is reached [55]. The critical domain size and activation energy for nucleation can be obtained from minimization of Eq. (9) as $r_{c}=0.83 b / a, l_{c}=1.86 b c^{1 / 2} a^{-3 / 2}$, and $E_{a}=$ $0.518 b^{3} c^{1 / 2} a^{-5 / 2}$, where $a=4 \pi P_{s} E / 3$. For typical ferroelectric materials such as $\mathrm{BaTiO}_{3}\left(\sigma=7 \mathrm{~mJ} / \mathrm{m}^{2}, P_{s}=0.26 \mathrm{C} / \mathrm{m}^{2}, \varepsilon_{11}=2000, \varepsilon_{33}=120\right.$ [41]) in the uniform field $E=10^{5} \mathrm{~V} / \mathrm{m}$ the corresponding values are $E_{a}=2.4 \cdot 10^{5} \mathrm{eV}$ and $l_{c}=16.4 \mu \mathrm{m}, r_{c}=0.264 \mu \mathrm{m}$. Thus, for relatively weak fields corresponding to experimental coercive fields, homogeneous domain nucleation is impossible, which explains why in typical ferroelectric materials domain nucleation occurs on the surface or at interface defects.

The opposite is true for the tip-induced switching, when the small radius of curvature of the tip results in large $\left(10^{6}-10^{9} \mathrm{~V} / \mathrm{m}\right)$ electric fields localized at the tip apex. The corresponding domain free energy can be determined from electroelastic 
field distribution generated by the PFM tip as

$$
\Delta G_{b u l k}=\int_{V} \Delta g_{b u l k}(\vec{r}) d V=2 \pi \int_{0}^{l_{d}} d z \int_{0}^{r(z)} \Delta g_{b u l k}(r, z) r d r,
$$

where $r(z)=r_{d} \sqrt{1-z^{2} / l^{2} d}$. The analysis of the switching process can be greatly simplified in the point charge model $[46,47]$ applicable if domain sizes $l_{d}, r_{d} \gg$ $R, a$, where $R$ is the tip radius and $a$ is the contact radius and provided that the singularity at the origin is weak enough to ensure convergence of the integral in Eq. (10). For ferroelectric switching induced by a point charge $q_{s}$ located on the surface, the integral in Eq. (10) can be taken analytically and $\Delta G_{b u l k}=d r_{d} l_{d} /\left(l_{d}+\gamma r_{d}\right)$, where $d=2 P_{s} q_{s} /\left(\varepsilon_{0}+\sqrt{\varepsilon_{11} \varepsilon_{33}}\right)$ and $\gamma=\sqrt{\varepsilon_{33} / \varepsilon_{11}}$. In this case, domain size and energy are $r_{e}=0.342 d^{2 / 3}(b c)^{-1 / 3}, l_{e}=0.2 d / b$, and $E_{m}=-0.205 d^{5 / 3}(b c)^{-1 / 3}$ $[46,47]$. The activation energy for domain nucleation in this approximation is zero, due to the infinite field at the origin. This analysis predicts that domain shape in the switching process follows the invariant relation $r_{e}^{3} / l_{e}^{2}=b / c$.

The applicability of the point-charge approximation to the thermodynamics of domain switching on the large length scales is limited by the contribution of the electrostatic fields produced by the conical part of the tip, which decay much slower than that produced by the point charge. At smaller length scales comparable to the tip radius of curvature, the thermodynamics of switching process requires an exact electroelastic field structure to be taken into account. The evolution of a free energy surface for macroscopic switching in a uniform field $\left(10^{5} \mathrm{~V} / \mathrm{m}\right)$ and point charge field are shown in Figure 7(a), (b). Free energy surfaces for several point-charge magnitudes and $h=10 \mathrm{~nm}$ are shown in Figure 7(c-e). For $q_{a}=100 \mathrm{e}^{-}$, the free energy is positive for all $l_{d}, r_{d}$ and a domain does not form. For $q_{a}=200 \mathrm{e}^{-}$ the free energy surface develops a kink. Finally, for $q_{a}=400 \mathrm{e}^{-}$the free energy minimum corresponding to a stable domain and a saddle point corresponding to the activation energy for nucleation are clearly seen. Finally, the free energy surface for a tip radius $R=50 \mathrm{~nm}$ and bias $V=5 \mathrm{~V}$ in the weak indentation regime is shown in Figure $7(f)$. Note that domain nucleation requires certain threshold bias of the ot order of $0.1-1 \mathrm{~V}$, corresponding to nonzero activation energy for nucleation (of order of $\sim k T$ ) [49].

In the last several years, a number of reports have become available on highorder ferroic switching in PFM $[5,25]$. Due to the rapid decay of corresponding electroelastic fields, the use of Eq. (13) in point-charge/force approximation results in singularity in the contact area, necessitating exact electroelastic field structure to be taken into account. Using rigorous electroelastic solutions, it was shown [48] that for higher-order ferroic switching (e.g., ferroelectroelastic), the domain size is limited by the tip-sample contact area, thus allowing precise control of domain size.

\subsection{Time-Dependent Measurements in PFM}

Applications of ferroelectric domain patterning for data storage, electrooptical devices, and ferroelectric lithography necessitate fundamental studies of the domain 

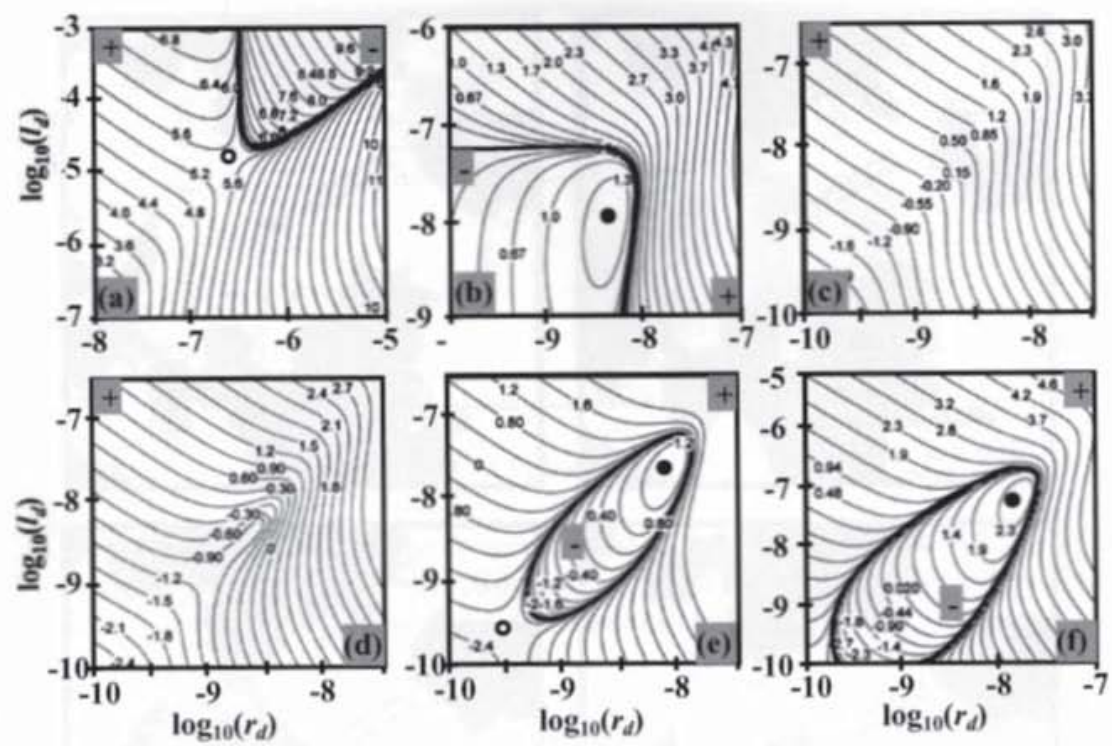

FIGURE 7. The free energy surface for domain switching in (a) uniform electric field and (b) field produced by a point charge on the surface and (c-e) above the surface. Relevant parameters are given in the text. (f) The free energy surface for a realistic tip shape. Plotted is the logarithm of the absolute value of the energy in eV. Solid lines separate regions of opposite signs, indicated by the plus and minus signs. Saddle points (o) and local minima (•) are shown. Reprinted with permission from [49]. Copyright 2005, American Institute of Physics.

switching process, including thermodynamics and kinetics of domain nucleation, growth, and relaxation. Although the high spatial resolution of PFM provides a unique opportunity for investigation of domain dynamics in thin films, its poor time resolution, which is determined by the time required for image acquisition (at the scan rate of $2 \mathrm{~Hz}$ and image resolution of $256 \times 256$ pixels, it takes about $2 \mathrm{~min}$ to acquire an image), makes the in situ measurements of domain dynamics during fast switching processes difficult. While PFM can be readily used to investigate slow polarization relaxation processes with characteristic times on the order of minutes and above, it is a great challenge to deduce the mechanism of ferroelectric domain transformation when polarization reversal occurs in a matter of microseconds and faster.

This problem can be partly circumvented by studying domain dynamics in a quasi-static regime using a so-called step-by-step switching approach developed and applied to PFM (see, e.g., [4]). In this approach, partial reversal of polarization is generated by applying a voltage pulse shorter than the time required for full switching but with the pulse amplitude fixed above threshold voltage. By applying a sequence of such short pulses of incrementally increasing duration with PFM domain imaging after each pulse, a consistent picture of domain dynamics can 

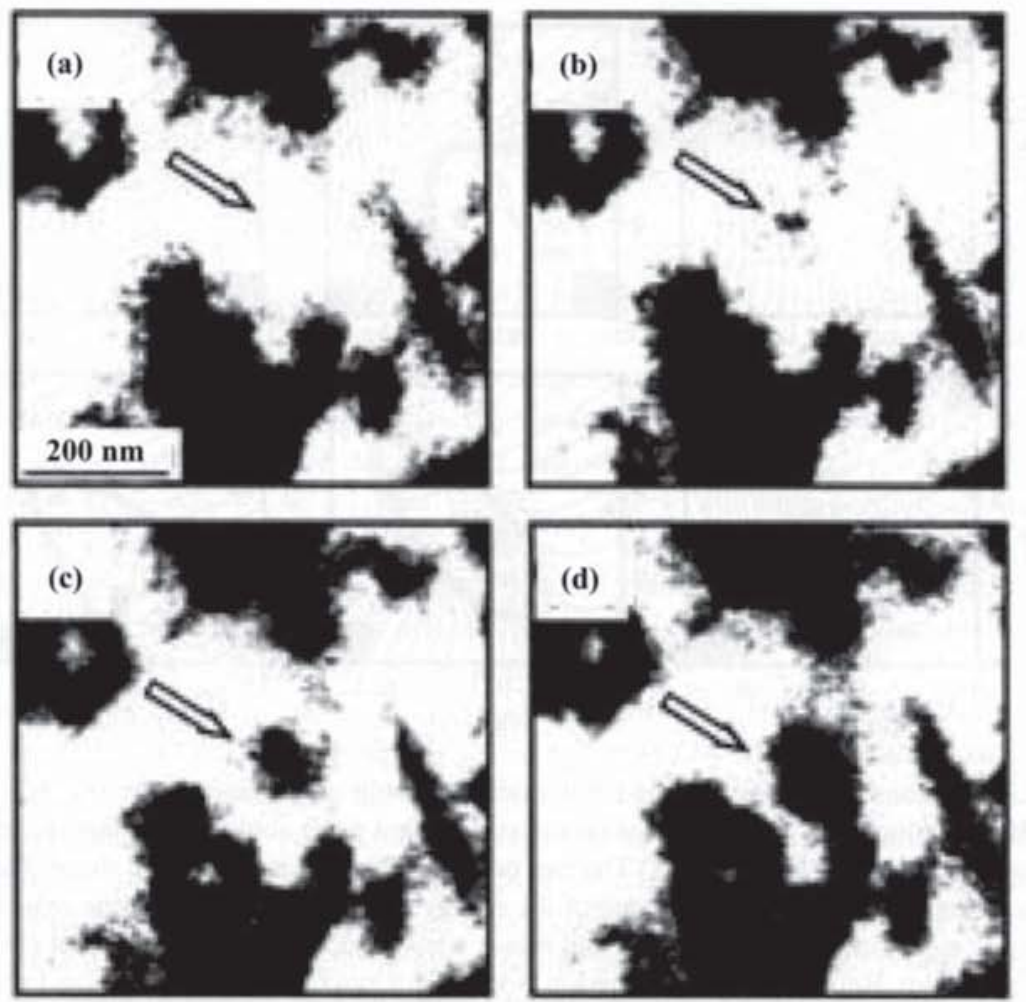

FIGURE 8. Sequence of PFM images of PZT film showing a sidewise growth of a domain in the center of the image. The growth is induced by application of $5 \mathrm{~V}$ voltage pulses of increasing duration to the same spot by a fixed probing tip. (a) Original domain structure; (b-d) domain images after application of voltage pulses. Pulse duration: (b) $0.25 \mathrm{~s}$, (c) $0.5 \mathrm{~s}$, (d) $1 \mathrm{~s}$. Reprinted with permission from [2]. Copyright 2004, American Scientific Publishers.

be obtained. This approach can provide information on the domain wall velocity, its spatial anisotropy, and its field dependence. To avoid data misinterpretation due to spontaneous back-switching between the pulses, stability of the produced intermediate patterns should be checked by acquiring domain images at different time intervals after single pulse application.

An example of this approach is illustrated in Figure 8, which shows a sequence of piezoresponse images of a growing domain in $\mathrm{Pb}(\mathrm{Zr}, \mathrm{Ti}) \mathrm{O}_{3}(\mathrm{PZT})$ films. Based on the contrast of the growing domain, it is assumed that the domain fully extends through the film thickness after the first pulse and further growth occurs via the sidewise expansion only. The diameter of the smallest stable domain produced by the first voltage pulse of $5 \mathrm{~V}$ varied from $20 \mathrm{~nm}$ to $40 \mathrm{~nm}$. Since the electric field generated by a probing tip quickly decreases with distance, the rate of domain growth, after fast initial expansion, slows down until the domain stops growing. To 


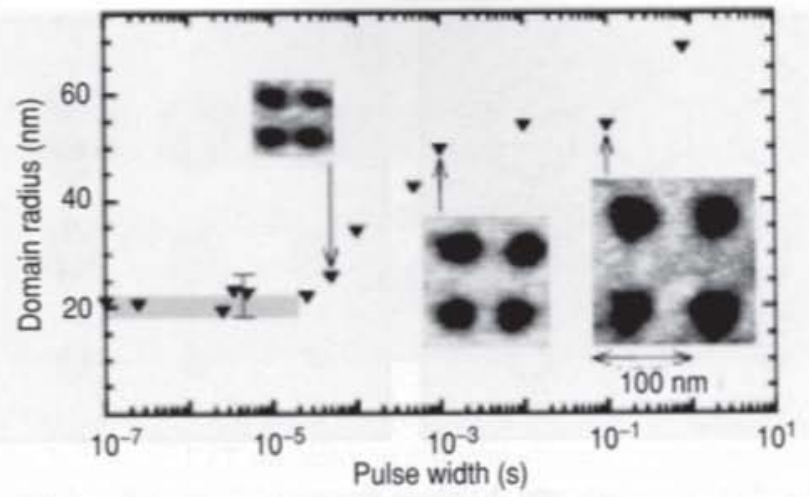

FIGURE 9. The dependence of the domain size on the switching time in $\mathrm{Pb}\left(\mathrm{Zr}_{0.2} \mathrm{Ti}_{0.8}\right) \mathrm{O}_{3}$ films of $29 \mathrm{~nm}$ thick. Insets show the corresponding images of scanned domains. Reproduced with permission from [36]. Copyright 2002, American Physical Society.

describe the sidewise expansion of the domain, it is necessary to take into account the field dependence of the domain wall velocity and the spatial distribution of the electric field generated by the probing tip.

In the step-by-step approach, the time resolution is determined by the pulse length increment, which can be in the nanosecond range. This is particularly important from the viewpoint of studying domain switching dynamics in thin-film ferroelectric capacitors used in memory devices where the switching time is typically below $100 \mathrm{~ns}$. In this case, the time resolution will also depend on the rise time of the input pulse generated by the voltage source and on the size of the capacitor, i.e. its $R C$-time constant [56,57].

\subsubsection{Domain Growth During Tip-Induced Switching}

The measurement of the size of switched domains versus the magnitude of applied voltage and time can give us insight into the mechanism of polarization switching in ferroelectric films. Although the PFM measurements are quite slow, it is possible to distinguish between different mechanisms that control the propagation of the domain wall in such a complex object as ferroelectric thin film with defects. Tybell et al. [36] by studying the time dependence of the size of switched domains by PFM tip could assign the motion of the domain wall to the creep mechanism. Figure 9 presents some of their results along with the images of switched domains in $\mathrm{Pb}\left(\mathrm{Zr}_{0.2} \mathrm{Ti}_{0.8}\right) \mathrm{O}_{3}$ epitaxial films prepared by magnetron sputtering on $\mathrm{SrTiO}_{3}: \mathrm{Nb}$ substrates that simultaneously served as a bottom electrode.

These measurements allowed calculations of the domain wall velocity as a function of the electric field. Within the limitation of the simple point-charge model, the results showed that even in a high-quality thin ferroelectric film the domain wall motion is limited by the creep mechanism with the critical exponent close to unity. In the subsequent work of the same group [58] these results were confirmed by the 

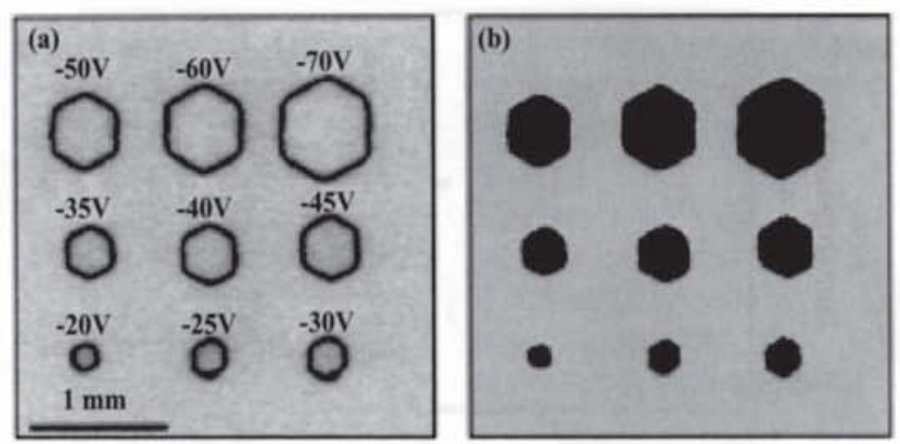

FIGURE 10. PFM (a) amplitude and (b) phase images of ferroelectric domains fabricated by 10 -ms voltage pulses of different amplitudes in $\mathrm{LiNbO}_{3}$ single crystals. Reprinted with permission from [59]. Copyright 2005, American Institute of Physics.

measurements of roughness of the domain walls, however, the critical exponent was found to be less than unity. Pinning defects limited domain wall propagation could not be identified in this work, however, the intrinsic pinning mechanism was ruled out. The results are useful for the development of high-density data storage devices.

In another work, Rodriguez et al. [59] studied domain switching kinetics in stoichiometric $\mathrm{LiNbO}_{3}$ single crystals by applying the voltage pulses of different amplitudes and durations over 5 orders of magnitude. The minimum size of the created domains was much larger than in the case of thin PZT films, and the mechanism of domain wall motion in this case was found to be different. Figure 10 demonstrates the written domains patterns obtained with poling under different voltages.

The domain wall velocity was calculated as a function of the domain radius and local electric field using distribution function defined by a charge sphere model. It was shown that at sufficiently large domain sizes, the motion of the domain wall is the electric-field activated process, while for the small sizes it is not activated since the applied electric field is much greater than the coercive field. The domain wall velocity $v$ was satisfactorily fitted to a simple equation $V(r) \propto$ $\exp \left[-\alpha / E\left(r+r_{0}\right)\right]$, thus confirming the time-dependent switching results. Here $\alpha$ is the activation field and $r_{0}$ is the fitting parameter. It was interpreted as the domain radius at which an activated process begins. As expected, $r_{0}$ increases with increasing applied voltage reaching $110 \mathrm{~nm}$ under $100 \mathrm{~V}$. Along with the width of the domain wall, high values of $r_{0}$ may limit the use of $\mathrm{LiNbO}_{3}$ single crystals for dense data storage.

\subsubsection{Domain Dynamics in Capacitor Configuration}

The PFM technique also allows investigation of the domain dynamics in a "normal," i.e., plane capacitor situation, where the electric field distribution is homogeneous across the thickness of the ferroelectric layer. In this case, the PFM 


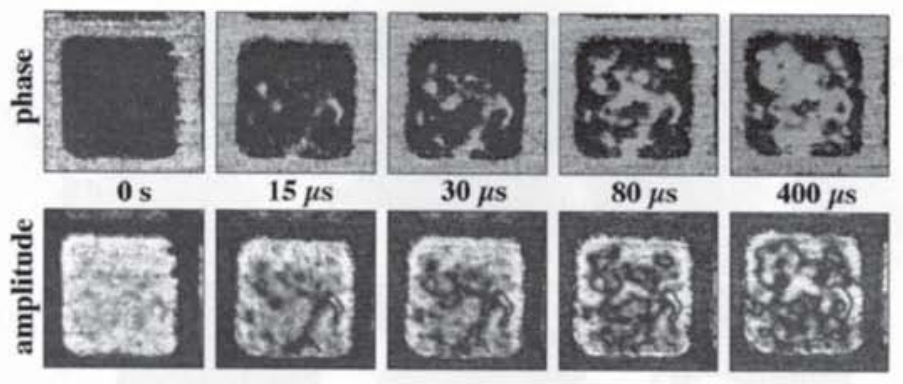

FIGURE 11. PFM phase and amplitude images of instantaneous domain configurations developing in the $3 \times 3 \mu \mathrm{m}^{2}$ PZT capacitor at different stages of polarization reversal under a 1.1 V bias. Reprinted with permission from [60]. Copyright 2004, American Institute of Physics.

results can be directly compared with the switching data obtained by conventional measurements. Direct studies of domain dynamics during polarization reversal in 180-nm-thick PZT capacitors have been performed by Gruverman et al. [60] (Figure 11). These results have been used to elucidate the switching mechanism in PZT capacitors. It has been shown that at low voltages a Kolmogorov-Avrami-Ishibashi mechanism [61] is applicable, while at longer times the broad distribution of the relaxation times should be taken into account. Thus, the PFM method can be used for direct experimental verification of the existing theoretical models of switching in a broad range of the switching times.

\subsubsection{Domain relaxation}

Along with the study of the mechanisms of the domain nucleation and growth, the PFM method is also applicable to the investigation of domain relaxation, i.e., the process always accompanying depolarization. The stability of the polarization state created by the external voltage is a prerequisite for the realization of SPMbased ferroelectric memories, since it directly controls retention time of the stored bits. In addition, polarization relaxation is important for the understanding of aging phenomena in piezoelectric ceramics. The advantage of PFM as compared to macroscopic measurements of depolarization is that it allows understanding this effect locally rather than by the averaging over many backswitching events. Unfortunately, the time resolution of PFM measurements is significantly smaller than in macroscopic measurements preventing studying initial stages of polarization loss.

Several studies have been recently performed on the relaxation phenomena by PFM. Despite these efforts, there is still a lack of reliable data on most of ferroelectric materials useful in device applications, including the time dependence of the retention loss in ferroelectric films. While some authors reported a log-linear dependence of the polarization relaxation in PZT films [62], other groups deduced a stretched exponential dependence [63] in the same material. Moreover, polarization relaxation has been found to differ in epitaxial films, where a spontaneous 


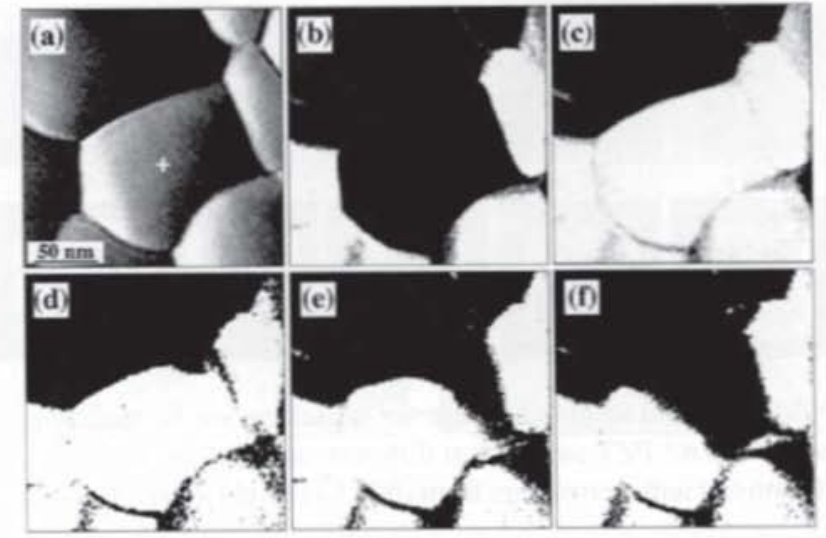

FIGURE 12. Retention loss in PZT(20/80) film on LSCO/TiN/Si measured after poling with $6 \mathrm{~V}$ applied for $0.2 \mathrm{~s}$. (a) Topographic image with cross indicating poling point, (b) as-grown domain structure, (c) domains immediately after poling, and (d-f) evolution of domain structure after 4, 90, and $140 \mathrm{~min}$ after poling. Reprinted with permission from [4]. Copyright 1998, Annual Reviews.

nucleation of $180^{\circ}$ domain walls on existing twin boundaries has been found to modify the relaxation process. [9] In this case, it was possible to use a modified Avrami-Kolmogorov kinetics [61] for the description of depolarization process in ferroelectric thin films. Figure 12 illustrates the polarization loss in PZT films studied by PFM [4]. A voltage pulse of $6 \mathrm{~V}$ was applied for $0.2 \mathrm{~s}$ in the center of the middle grain and dynamics of domain relaxation was observed during subsequent scanning. As can be seen the domain relaxation proceeds mainly through the sidewise motion of the domain walls.

It has been found that the domain walls measured in dynamics appear much thicker than in static conditions. This is a natural result caused by the intrinsic disadvantage of PFM: slow scan rate. From a quantitative analysis of domain images, it follows that the domain wall slows down with time, giving rise to a familiar logarithmic dependence of the fraction of reversed domains: $P(t)=P_{0}-m \log \left(t / t_{o}\right)$. As usual, the log time dependence suggests a broad distribution of relaxation times in polycrystalline ferroelectric films. Note that the shape of the shrinking domain becomes irregular due to the spatial variation of domain wall velocity. PFM retention measurements in SBT films [64] were found to be highly asymmetric, i.e., the application of positive voltage pulses gave much better stability of domain structure as compared to negative ones. Such asymmetric retention (called generally an imprint) was found also in macroscopic measurements. On the other hand, the same SBT films grown on $\mathrm{RuO}_{2}$ electrodes showed no sign of polarization retention, irrespectively of the sign of applied voltage. Based on these experiments. Gruverman and Tanaka [64] developed a qualitative model of polarization retention in SBT-type materials. 


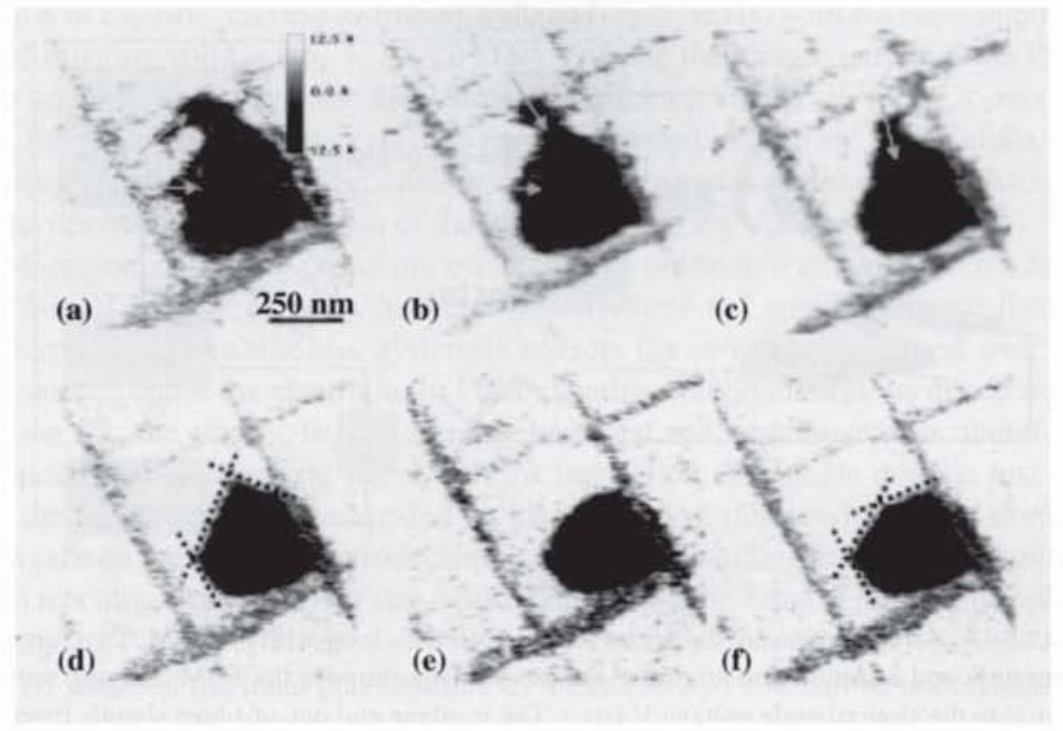

FIGURE 13. (a-f) Relaxation of the domains in epitaxial PZT films after poling during scanning. Arrows indicate the direction of the velocity of moving wall in areas with high curvature. Faceting is shown in the final stage of domain relaxation (dotted lines). Reprinted with permission from [65]. Copyright 2001, American Physical Society.

In another experiment [65], the authors studied the polarization relaxation via PFM in epitaxial $\mathrm{PbZr}_{0.2} \mathrm{Ti}_{0.8} \mathrm{O}_{3}$ films on $\mathrm{LSCO}$ electrodes. These films were found to have a large number of $90^{\circ}$ domains (twins) that strongly contributed to domain wall relaxation. Figure 13 gives an example of local depolarization process in such films.

The essential feature of such relaxation observed after poling under scanning with a dc bias applied to the tip is that the back-switching mainly starts near the existing $90^{\circ}$ domain wall and propagates with a velocity that depends on the curvature of domain wall. In the final stage of domain relaxation, faceting of the domain wall is seen that is a result of slowing down the relaxation along certain crystallographic directions. Investigation of the relaxation of such domain walls opens up new horizons for the understanding domain-wall propagation in such complicated objects as anisotropic ferroelectrics with defects.

\subsection{Local Piezoelectric Hysteresis Loops}

Along with the sequental polarization switching performed with a series of voltage pulses applied to the tip followed by consequent imaging, PFM can be used in a spectroscopy mode when the measurements are done on a fixed tip position (local $d_{33}$ acquisition) under a dc voltage swept in the cyclic manner. This dependence of the local piezoelectric vibration on applied bias is referred to as a 


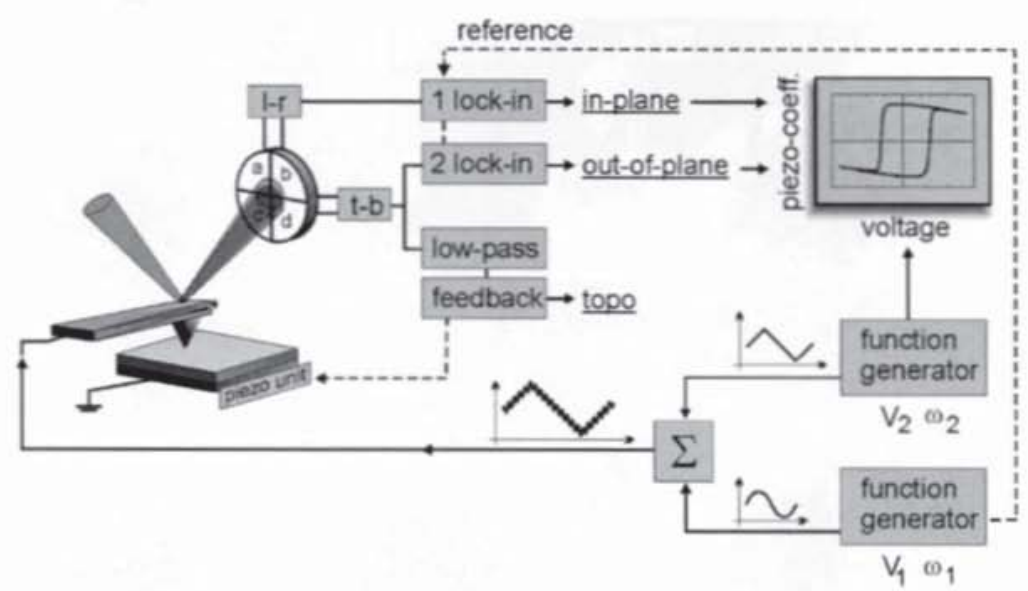

FIGURE 14. Setup to measure the piezoelectric hysteresis loop with the PFM. Two function generators and a summation amplifier are used to superimpose the PFM imaging voltage $V_{1}\left(\omega_{1}\right)$ to the slow triangle voltage $V_{2}\left(\omega_{2}\right)$. The in-plane and out-of-plane signals from the PFM are plotted in an oscilloscope versus the switching voltage $V_{2}$ to obtain the piezoelectric hysteresis loops.

piezoelectric hysteresis loop. If performed on a macroscopic scale, this loop corresponds to a weak-field piezoelectric coefficient tuned by a continuously varying bias field. According to a linearized electrostriction equation, the piezoelectric coefficient can be often expressed as $d_{33}=2 Q \varepsilon_{33} P_{3}$, where $Q$ is the longitudinal electrostriction coefficient and $\varepsilon_{33}$ and $P_{3}$ are the corresponding dielectric constant and spontaneous polarization values. Therefore, the $d_{33}$ variation reflects polarization switching with corresponding tuning of the dielectric permittivity $\varepsilon(\mathrm{E})$ and polarization $P(E)$, which both affect piezoelectric coefficient [66]. Piezoelectric hysteresis loops are indeed very useful for the identification of the switching mechanism and ferroelectric degradation both in thin film [67] and bulk ferroelectrics [68]. It should be stressed that at the nanoscale level the shape of piezoelectric loops is different [69], reflecting different physical mechanisms involved. Measurements of local hysteresis loops are of great importance in inhomogeneous or polycrystalline ferroelectrics because they are able to quantify polarization switching on a scale significantly smaller than the grain size or inhomogeneity variation (typically few tens of $\mathrm{nm}$ ).

Figure 14 depicts a typical setup to measure the piezoelectric hysteresis loop by PFM. In addition to the conventional PFM configuration (Figure 2), a second function generator, a summing amplifier, and an oscilloscope are needed. The first function generator provides the PFM imaging voltage $V_{1}\left(\omega_{1}\right)$, and the second function generator supplies the switching voltage $V_{2}\left(\omega_{2}\right)^{5}$. Adding these two signals

${ }^{5}$ Here a saw voltage variation is used to initiate switching, as this is the standard waveform for macroscopic hysteresis measurements. 
results in a slowly varying switching voltage $\left(\omega_{2} \gg \mathrm{mHz}\right)$ with the superimposed PFM imaging voltage $\left(\omega_{1}>10-20 \mathrm{kHz}\right)$. Feeding the lock-in output of the PFM into an oscilloscope (y-axis) and using the switching voltage $V_{2}$ as the $x$-axis input, the piezoelectric hysteresis loop can be recorded. Acquiring both signals, the in-plane and the out-of-plane, enables one to obtain two complementary hysteresis loops describing the evolution of the piezoresponse signal upon switching.

Macroscopically, the switching occurs via the nucleation and growth of a large number of reverse domains in the situation where the applied electric field is uniform. Therefore, the $d_{33}$ hysteresis reflects the switching averaged over entire sample under the electrode. In PFM experimental conditions, as discussed in section 3.1, the electric field is strongly localized and inhomogeneous, therefore, the polarization switching starts with the nucleation of a single domain just under the tip [70]. When the applied dc bias increases, this newly formed domain elongates to the bottom electrode, simultaneously expanding in lateral dimensions until reaching an equilibrium size, which depends on the value of maximim applied voltage.

The shape of the local piezohysteresis measured by PFM can be understood by scanning the area adjucent to the tip in-between the voltage increments, provided no significant relaxation occurs. In addition, useful information on the propagation of domain walls in ferroelectrics can be obtained. An example of the vizualization of the piezoelectric hysteresis in polycrystalline PZT films prepared by sol-gel is shown in Figure 15 [71]. The measurements were done by the setup shown in Figure 14, but the switching voltage was periodically interrupted for scanning. The images are shown near the corresponding points of the hysteresis loop.

It is seen that the sudden contrast change (arrow at $V^{*}$ ) corresponds to the appearance of the stable inverse domain. Thus nucleation voltage $V^{*} \approx 11 \mathrm{~V}$ can be considered as an analog of the coercive voltage in local measurements rather than the voltage where $d_{33}=0$. A rapid increase of $d_{33}$ with increasing $V_{\mathrm{dc}}$ is explained by the forward and lateral growth of the nucleated domain, progressively contributing to the PFM signal. When the size of the inverse domain is much greater than the penetration depth of the weak ac field, the tip senses only a fully polarized area with aligned polarization and $d_{33}$ is well saturated. Figure 15 also illustrates the propagation of domain wall in polycrystalline ferroelectrics where local inhomogeneities and stresses make the domain boundary strongly irregular.

It is instructive to compare local piezoelectric hysteresis with that obtained in macroscopic configuration (homogeneous electric field). Figure 16 represents macroscopic $d_{33}$ loop (measured by laser interferometry as a deflection of metal electrode of macroscopic dimensions under the same combination of $\mathrm{dc}$ and ac voltages) in comparison with the local one for $\mathrm{PbZr}_{0.45} \quad \mathrm{Ti}_{0.55} \mathrm{O}_{3}$ films prepared by sol-gel method [69]. Local $d_{33}$ is given in arbitrary units as the calibration procedure (described in section 2.2.3) has not been performed.

This result demonstrates that both the shape of the loop and values of the coercive voltage are strongly affected by the measurement conditions. A notable decrease of the piezoresponse after saturation in the case of local measurements is caused by much greater depolarizing field in PFM conditions due to the influence of low- 


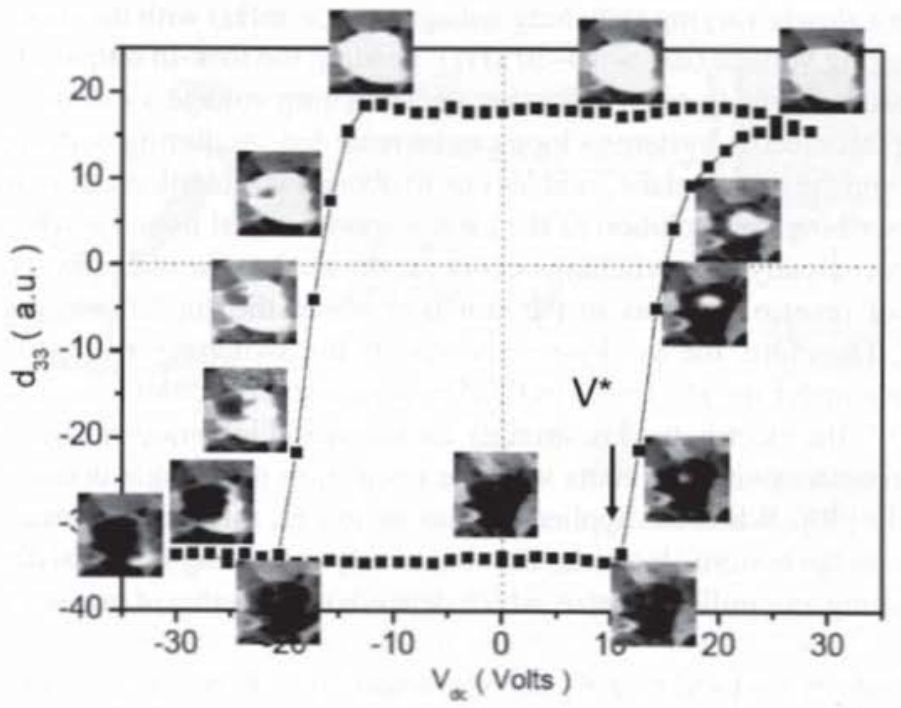

FIGURE 15. PFM hysteresis loop of thick PZT films prepared by sol-gel. The insets (scan size $1 \mu \mathrm{m}$ ) show the static domain structures obtained by scanning with the ac voltage of the amplitude of $1 \mathrm{~V}$ in the corresponding points of the hysteresis (adapted from [71]).

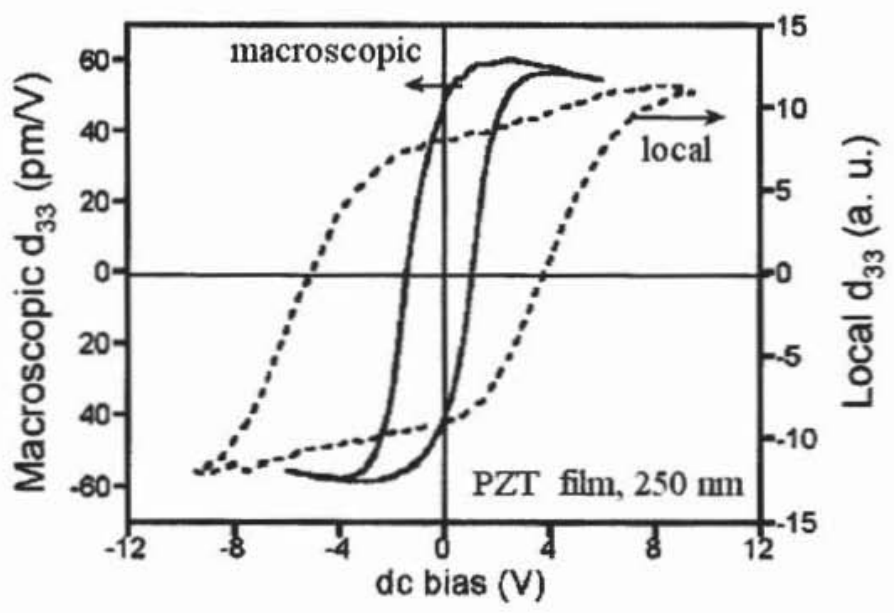

FIGURE 16. Comparison of macroscopic (interferometry) and local piezoelectric hysteresis loops measured by PFM (adapted from [69]).

dielectric-constant layer on the surface. On the other hand, the "coercive voltage" (zeroing of $d_{33}$ ) is much smaller in macroscopic conditions illustrating its different physical meaning.

The crystallographic orientation dependence of hysteresis loop was studied by Kalinin et al. [70]. Comparison of the film surface topography (Figure 17(a)) 

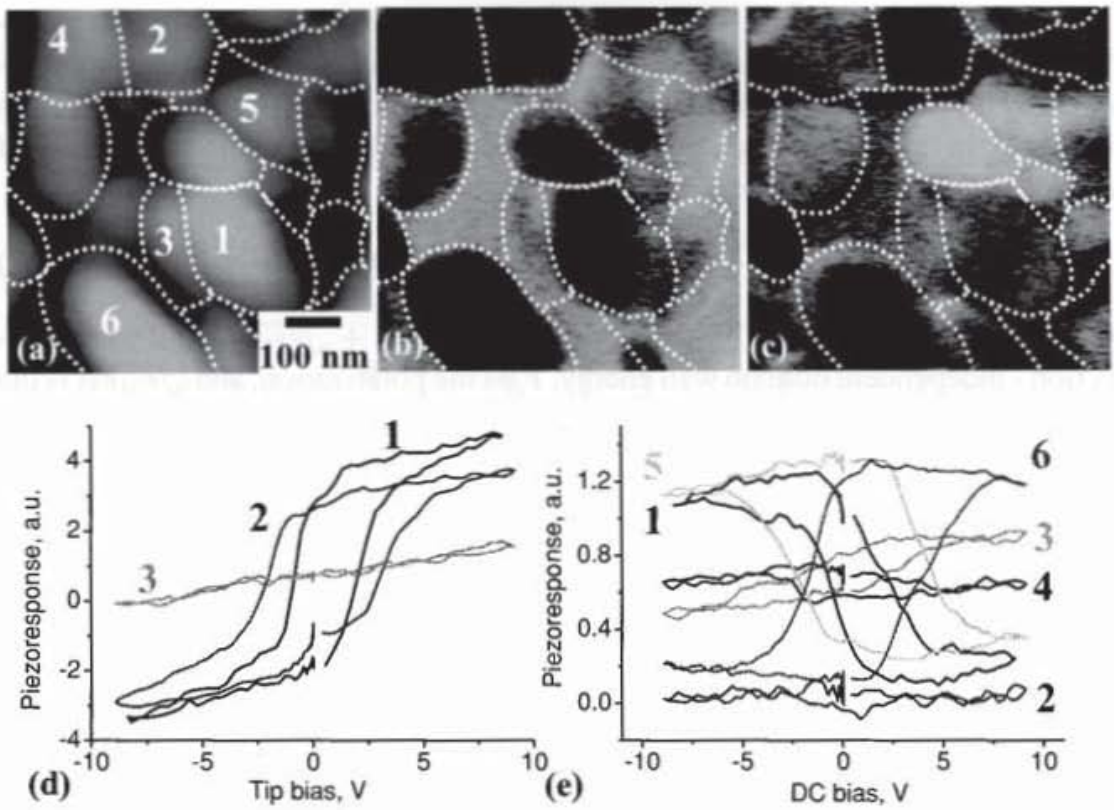

FIGURE 17. Sample surface topography (a), vertical (b), and lateral (c) PFM signal of SBT thin film. Vertical (a) and lateral (b) piezoelectric hysteresis loops for selected grains. For clarity, vertical loops are shown only for grains 1,2 , and 3 . Reprinted with permission from [70]). Copyright 2005, American Institute of Physics.

with vertical (Figure 17(b)) and lateral (Figure 17(c)) PFM images (VPFM and LPFM, respectively) suggests that most grains exist in a single domain state. Grains that exhibit strong VPFM contrast also have distinctive hysteresis loops (Figure 17(d)), indicative of a large out-of-plane polarization component. For grain 3, the VPFM hysteresis loop is linear, indicative of a non-ferroelectric nature of the grain or purely in-plane polarization corresponding to (001) orientation [72]. Complementary LPFM hysteresis loops of individual grains are shown in Figure 2(e). Unlike the vertical PFM hysteresis loops, lateral loops can be oriented in both directions depending on the in-plane polarization orientation with respect to the cantilever axis. This results in higher variability of the LPFM loops. Comparison of the angular dependence of piezoresponse signal and coercive bias suggests that for the off-axis orientation of the grains the response decreases and coercive bias increases, as can be expected from simple energy arguments. In the limiting case of the (001)-oriented grain piezoresponse and coercive bias become zero and infinity, respectively.

The analysis of the hysteresis loop shape can be performed in the thermodynamic $[70,73]$ and kinetic limits [74]. In the thermodynamic case, the domian size is equal to the thermodynamically limited size as analyzed in section 3.1 . In the kinetic limit, the domain size is limited by the velocity of the forward and lateral growth of the domain walls. 
Domain switching in the thermodynamic limit can be analyzed using the pointcharge model developed by Molotskii [47]. The electric field of the tip is approximated by the point charge on the smaple surface, coinciding with strong indentaiton limit [75]. In this case, the length of domain $l$ (perpendicular to the free surface of a ferroelectric) is proportional to the applied voltage:

$$
l=d / 5 b,
$$

where $b=\sigma_{\text {wall }} \pi^{2} / 2$ and $d=d_{0} V_{d c}=2 P_{s} C_{c} V_{d c} /\left(\varepsilon_{0}+\sqrt{\varepsilon_{11} \varepsilon_{33}}\right) . \sigma_{\text {wall }}$ is the direction - independent domain wall energy, $P_{s}$ is the polarization, and $\sqrt{\epsilon_{11} \epsilon_{33}}$ is the dielectric constant of the material. If we consider an intermediate situation (new domain does not reach bottom electrode), the measured piezoresponse will consist of two contributions having opposite sign: from the newly formed domain and from the remaining nonswitched part of the rest of the sample using a 1D model [76]. The shape of the hysteresis loop in PFM is given by $P R=d_{e f f}\{V(0)-2 V(l)\}$, where $V(0)$ is the potential at the surface and $V(l)$ is the potential at the domain boundary below the tip, and $d_{e f f}$ is the effective electromechanical response of the material [48]. It follows that the measured local piezoelectric coefficient equals zero not under the coercive voltage as in macroscopic conditions but simply when the contribution from the top domain cancels out the contribution of unswitched area $[V(0)=2 V(l)]$.

In the strong indentation regime, potential decays inversely with distance as $V(l)=\beta V(0) \alpha / l$, in which $\beta$ is a proportionality coefficient on the order of unity reflecting the field distribution in the material, $l$ is the distance from the center of the contact area, and $a$ is the contact radius. In the strong indentation limit, the capacitance of the tip-surface junction is $C_{c} \approx 4 a \sqrt{\varepsilon_{11} \varepsilon_{33}}$, thus yielding $d_{0} \approx$ $8 a P_{s}$. It follows that the tail of a single branch of the hysteresis response can be simply described by Equation:

$$
P R=d_{e f f}\left\{1-2 \beta a / l\left(V_{d c}\right)\right\} .
$$

In this thermodynamic limit, the shape of the hysteresis loop is determined solely by the domain wall energy and spontaneous polarization. Note that in the complete absence of pinning, the thermodynamic model suggests that the hysteresis will proceed along the solid line in Figure 18(a), i.e., the process is reversible and the domain should grow and shrink instantaneously in response to tip bias (Figure 18 (c)). However, if a weak pinning is included in the model, the actual domain wall boundary will lag behind that predicted by Eq. (12), and nucleation of a new domain will dominate the hysteresis loop, as illustrated in Figure 18(d). The saturation and remanent responses in this approximation are equal, and the onset of switching corresponds to the domain nucleation below the tip $\left(V_{c}^{+}=V_{c}^{-}=0\right.$ in the point charge limit when the field below tip is infinite).

From Eq. (12), the magnitude of the coercive bias can be derived as $V^{+}=V^{-}=$ $5 \beta \sigma_{\text {wall }} \pi^{2} / 8 P_{s}$ and for $\mathrm{BaTiO}_{3}\left(\sigma=7 \mathrm{~mJ} / \mathrm{m}^{2}, P_{s}=0.26 \mathrm{C} / \mathrm{m}^{2}\right)$ [74] the estimated coercive bias is $V^{+}=0.166 \mathrm{~V}$ (for $\beta=1$ ). Note that while Eqs. (11) and (12) are 


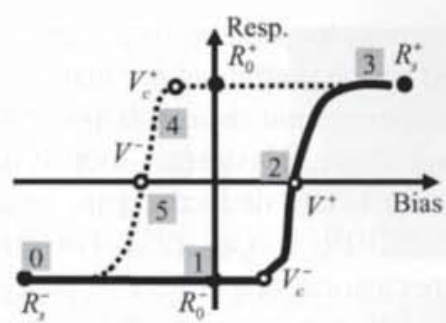

(a)

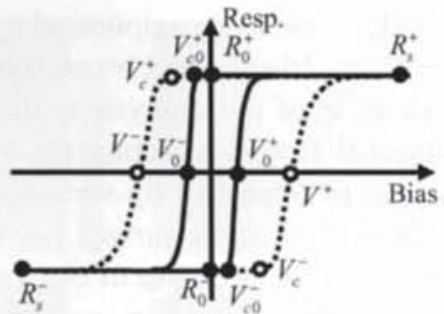

(b)

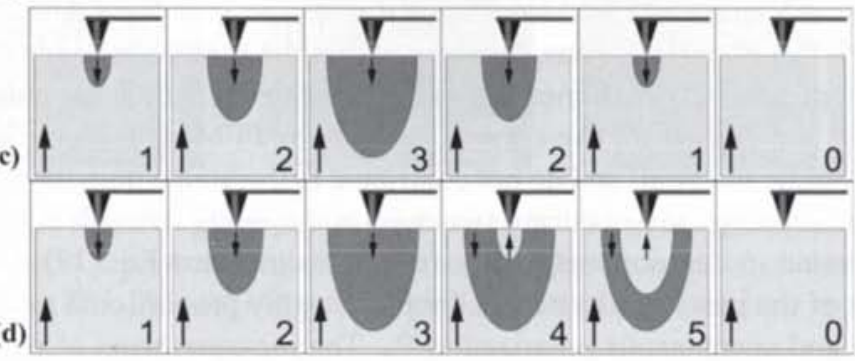

FIGURE 18. (a) Electromechanical hysteresis loop in the no pinning (solid line) and weak pinning (dotted line) limits. (b) Hysteresis loop (solid line) in the thermodynamic and kinetic (dotted line) limits. Forward and reverse coercive voltages, $V^{+}$and $V^{-}$, nucleation voltages, $V_{c}^{+}$and $V_{c}^{-}$, and forward and reverse saturation and remanent responses, $R^{+}, R^{-}, R_{s}^{+}$, and $R_{s}^{-}$, are shown. The effective work of switching $A_{s}$ is defined as the area below the loop. The imprint bias and maximum switchable polarization are defined as $\operatorname{Im}=\left(V^{+}+V^{-}\right) / 2$ and $R_{m}=R_{s}^{+}-R_{s}^{-}$, correspondingly. (c) Schematic representation of domain growth for the thermodynamic model without pinning and (d) with pinning. The numbers correspond to points on the hysteresis curve in (a). Arrows indicate domain orientation.

derived in the 1D approximation, in the general case, the effective piezoresponse is zero for some fixed domain size, $l_{\text {crit }}$, for which the response from the nascent domain below the tip and the surrounding material are compensating each other, giving rise to similar behavior.

However, the thermodynamic analysis ignores the effects related to the finite velocity of the domain wall required to explain time dependences in section 3.2 . In this kinetically limited process, the hysteresis loop broadens compared to thermodynamic case (Figure 18(b)). Experimentally, domain switching in ferroelectric materials has been shown to follow the phenomenological dependence $r(V, t)=k V$ Int, and domain wall velocity is thus $\dot{r}=k_{s} V / t$, where $k_{s}$ is the kinetic constant related to the pinning strength in the material [59]. Note that, while the kinetic data was obtained for the lateral domain size, the time dependences for vertical and lateral sizes can be expected to be commensurate.

In the spectroscopic PFM measurements, the envelope of the tip bias is ramped linearly with time, $V=b t$. Hence, $\dot{r}=k_{s} b$ and $r=k_{s} b t=k_{s} V$. Therefore, the domain size is independent of the ramp velocity and is determined by the pinning strength of the material alone. Thus, we recover the functional form of Eq. (12), 
which provides a robust description of hysteresis loop shape. The coercive bias is now $V^{+}=V^{-}=2 \beta a / k_{s}$, and is controlled by the pinning in the material.

With extension of this analysis to the experimental data, it is possible to analyze additional factors affecting the loop shape. Hysteresis loop broadening can occur due to imperfect tip-surface contact, i.e., dielectric gap, resulting in the attenuation of effective surface potential, $V(0)=\alpha V_{t i p}$ [23]. This gap effect will also result in the decrease of electromechanical response, $P R=d_{\text {eff }} / \alpha$. For frequency-independent dielectric constant of the gap, $\varepsilon_{d c}=\varepsilon_{\omega}$, the product of coercive bias and saturated electromechanical response is independent of the gap effect, $R^{+} V^{+}=$const $(\alpha)$. Thus, the area within the hysteresis loop (rather than loop width) provides a robust measure of the pinning strength in the material. The electrostatic and electrostrictive contributions to the PFM signal are conservative (for linear elastic material) and do not contribute to the area within the loop. Thus, the measure of energy losses during switching and pinning strength in the material are all included in the loop area. Also, from the simplified Eq. (12) we can find that the tilt of the measured hysteresis loop is roughly proportional to the applied dc voltage and spontaneous polarization $\mathrm{P}_{s}$. The measurements of piezoelectric hysteresis in PZT films of different compositions [73] confirmed that the slope of PFM hysteresis is really a function of the $\mathrm{P}_{s}$ value, thus giving a possbility of evaluating local ferroelectric properties.

Following progress continuous in hysteresis loop measurements by PFM, a technique referred to as switching spectroscopy PFM (SS-PFM), was developed recently by the ORNL group [77]. In SS-PFM, the local hysteresis loop is acquired at each image point, yielding a 3D data array. The relevant switching characteristics such as coercive biases, imprint, switchable polarization, and effective work of switching as defined in Figure 19 are extracted and plotted as 2D maps.

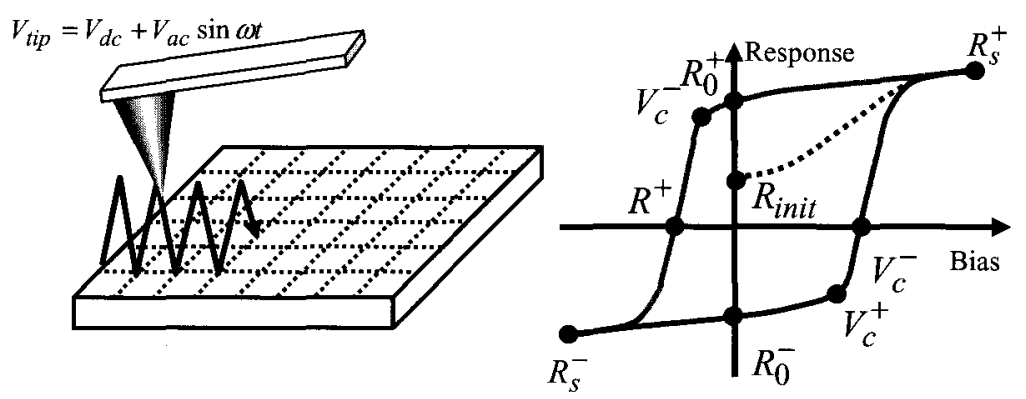

FIGURE 19. (a) In SS-PFM, the local hysteresis loop is collected at each point on $N x M$ mesh. (b) The single-point probing waveform in SS-PFM and data acquisition sequence. Forward and reverse coercive voltages, $V_{0}^{+}$and $V_{0}^{-}$, nucleation voltages, $V_{c 0}^{+}$and $V_{c 0}^{-}$, and forward and reverse saturation and remanent responses, $R_{0}^{+}, R_{0}^{-}, R_{s}^{+}$, and $R_{s}^{-}$, are shown. The work of switching $A_{s}$ is defined as the area within the loop. The imprint bias and maximum switchable polarization are defined as $\operatorname{Im}=\left(V_{0}^{+}+V_{0}^{-}\right) / 2$ and $R_{m}=R_{s}^{+}-R_{s}^{-}$ correspondingly. 

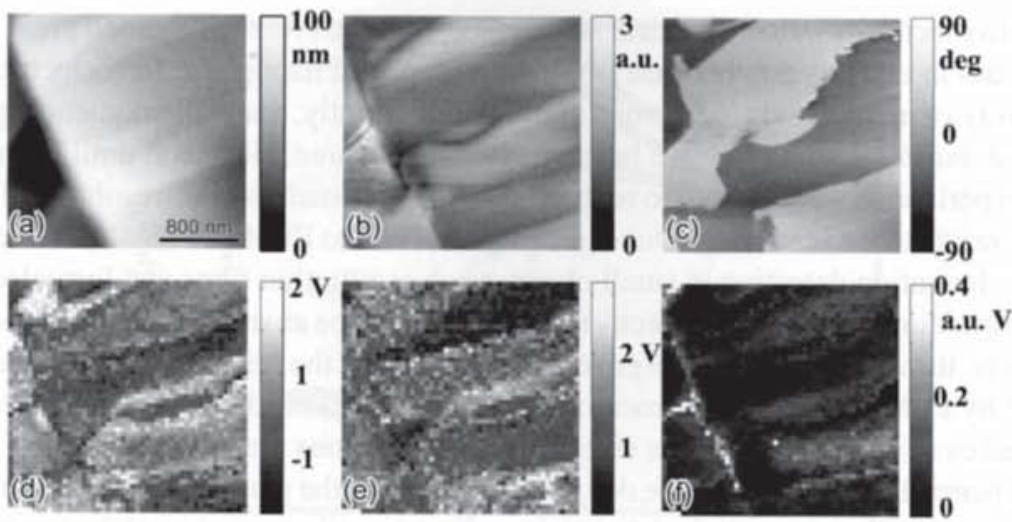

FIGURE 20. (a) Surface topography, piezoresponse (b) amplitude and (c) phase and SSPFM (d) imprint, (e) hysteresis loop width, and (f) effective work of switching maps of polycrystalline PZT. Note the enhanced pinning in the vicinity of the grain boundary.

The operation principle of SS-PFM and several imaging examples are shown in Figure 20.

\section{Applications of PFM}

Since the middle of 1990 s, the PFM method has been developed by several groups, giving rise to an ever-increasing number of publications ( $>120$ publications in 2004). This method is becoming the most popular tool in the science and technology of ferroelectrics and is considered as a main instrument for getting information on ferroelectric properties at the nanoscale. Below we briefly review recent activities on nanoscale studies in various ferroelectric systems, focusing on the application of PFM for solving fundamental and technological problems.

\subsection{Size Effects in Ferroelectrics}

Recent advances in the science and technology of ferroelectrics have resulted in ferroelectric materials and devices with nanostructures with length scales on the order of or less than several hundred nanometers [78]. Typical examples include, but are not limited to, thin films for dynamic random access memories, microactuators and $\mathrm{rf} / \mathrm{microwave}$ phase shifters, to name a few. Ferroelectrics have also become a candidate gate-dielectric material for field effect transistor applications due to their high permittivity. As device dimensions are scaled down, a size range is reached where ferroelectric materials show pronounced size effect. Size effects in ferroelectrics lead to a significant deviation from bulk properties, which is a consequence of a size-driven instability in the polar phase followed by the suppression 
of ferroelectricity below a critical size. Research in this field has gained great momentum in recent years because of the importance of nanophase ferroelectrics in an array of applications mentioned above. Concurrently, many phenomenological and $a b$ initio calculations have been directed to this area. However, until recently, the experiments were limited to scattering techniques such as high-resolution TEM or X-ray/neutron scattering. Direct techniques, such as PFM, are ideally suited for the polarization detection in small objects such as ultrathin films and ferroelectric nanostructures because the effective tip diameter can be as small as few nanometer. Clearly, the assessment of size-governed evolution of the ferroelectric state can be done by PFM under the influence of electric filed and mechanical forces. Recent advances of using PFM for the size effect investigations are outlined below.

In general, size effects can be defined as changes of the material properties due to the change of their geometrical dimensions. Keeping all parameters of the materials constant, just shrinking its size can result in a variety of different characteristics. Following parameters were found to change in ferroelectrics as their size decreases: (i) reduction of the remanent polarization, dielectric permittivity, and, consequently piezocoefficient; (ii) increasing coercive field; (iii) change in the domain structure; (iv) lower phase transition temperature and smearing of the phase transition.

All these features can be, in principle, assessed by PFM if the measurements are properly performed. Many theoretical approaches have been used to calculate and to predict the critical size or thickness for ferroelectricity. For example, using a mean-field Ginzburg-Landau approach, the free energy of the finite system, including volume and surface terms, was calculated and minimized. [79]. These and other calculations revealed a material-dependent critical thickness and correlation volume below which ferroelectric is loosing its polar nature. Using this approach, a critical thickness of $20 \mathrm{~nm}$ at room temperature has been predicted for PZT films. However, recent ab initio calculations [80] were able to show that, with appropriate boundary conditions, ferroelectricity can occur at the monolayer level.

Using the most advanced structuring techniques like electron-beam lithography, e-beam direct writing, or focused-ion-beam etching, the smallest achievable ferroelectric structures were fabricated to study the intrinsic size effects. However, the majority of reported size effects in the devices structured from thin films can be attributed to a reduction of the in-plane strain and the influence of the structuring method has to be taken into account, e.g., damage from etching, doping with sputter ions, etc. A straightforward method to fabricate a ferroelectric capacitor was introduced by Stanishevsky et al. [81], using focused-ion-beam equipment to "cut out" the device from an electrode/ferroelectric/electrode heterostructure. Different samples were prepared by varying the milling depth. To fabricate a capacitor structure, the multilayer was milled either to the ferroelectric layer (only the top electrode was structured) or down to the bottom electrode. By optimizing the process it was possible to achieve $\mathrm{Pt} / \mathrm{SBT} / \mathrm{Pt}$ capacitors of $100 \times 100 \mathrm{~nm}^{2}$ and $\mathrm{Pt} / \mathrm{LSCO} / \mathrm{PNZT} / \mathrm{LSCO} / \mathrm{Pt}$ capacitors of $70 \times 70 \mathrm{~nm}^{2}$ [82,83]. Even for the smallest capacitors, no intrinsic size effects were detected by PFM. For capacitors milled down to the bottom electrode a higher piezoelectric response was measured by PFM compared to capacitors where only the top-electrode was structured 


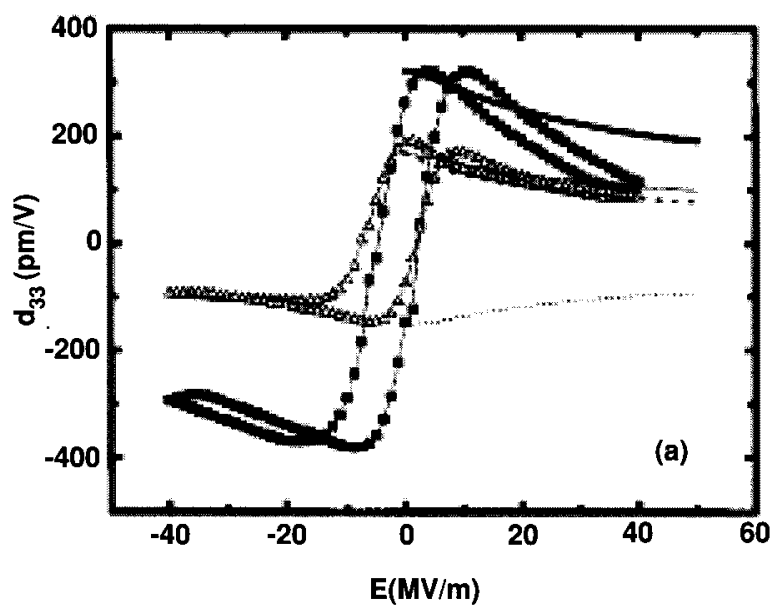

FIGURE 21. Piezoelectric hysteresis measured via PFM for PZT 50/50 film 200-nm thick. Solid symbols are used for a patterned $500 \times 500-\mathrm{nm}$ capacitor, while open symbols denote hysteresis for unpatterned film. Reprinted with permission from [82]. Copyright 1999, American Institute of Physics.

(Figure 21). This result was correlated to the in-plane constraints in the continuous film, rather than to intrinsic size effects. The hystereis loops obtained by PFM in PZT capacitors of different dimensions. No change of the intrinsic properties were detected except of the reduction due to clamping effect of the substrate. Similar results were obtained by Bühlmann et al. [84]. They deposited an epitaxial PZT thin film and used e-beam lithography to fabricate a $\mathrm{Cr}$ hard mask by means of a lift-off process. Subsequent reactive ion etching (RIE) led to ferroelectric island of sizes from $200 \times 200 \mathrm{~nm}^{2}$ down to $100 \times 100 \mathrm{~nm}^{2}$. The piezoelectric response showed a strong increase with decreasing feature size. They explained the increase of the piezoelectric response by a decreasing fraction of $a$-domains for the PZT islands, whereas the continuous film exhibited $15 \%$ of $a$-domains, which again is correlated to different mechanical strain.

A direct electron-beam writing technique (EBDW) to achieve a submicron metal pattern was introduced by Craighead and Schiavone [85]. First, an organometallic polymer thin film was locally irradiated with an electron beam, rendering the exposed area insoluble in an organic solvent. In a second step the unexposed areas were removed with a solvent. A heating process is added to remove the organic material and leave the metal pattern. Enhancing the EBDW method, Alexe et al. [86] could fabricate SBT and PZT structures as small as $100 \times 100 \mathrm{~nm}^{2}$. The coercive field was found to be size-independent. Contrary to the above-referenced papers, the authors of [86] reported a decrease of the piezoelectric response with decreasing size.

While studying size effects in devices structured from thin films, one of the biggest challenges is to distinguish between effects caused by the fabrication 
method, i.e., damage or contamination of the surface layer of the ferroelectric and the intrinsic size effects. The electron beam direct writing is an exceptional method were no obvious damage or contamination is expected to occur. Nevertheless, like the other structuring methods, no intrinsic changes in the ferroelectric properties can be observed down to the achievable device sizes.

Another technique frequently used to fabricate ferroelectric nanostructures is self-assembly. In this case, less damage is expected, and the minimum feature size can be as small as a few nanometer. Seifert et al. [87] and Fujisawa and coworkers [88] studied the growth of ferroelectric thin films in the early stage of a deposition process. The smallest PZT and $\mathrm{PbTiO}_{3}$ islands investigated by Fujisawa were $80 \mathrm{~nm}$ in diameter and exhibited a clear piezoelectric hysteresis loop without notable size effects.

Self-assembly approaches use the microstructural instability observed by Seifert et al. for the epitaxial growth relationship between very thin $\mathrm{PbTiO}_{3}$ films and $\mathrm{SrTiO}_{3}$ single crystalline substrates. It led to the formation of single-crystal islands instead of continuous films [87]. The normally undesirable process was adapted to silicon substrates with platinum bottom electrodes in a tailored way to generate separated 3D nanograins [89]. In this way, highly diluted $\mathrm{PbTiO}_{3}$ precursor solutions were deposited onto $\mathrm{Pt}(111) / \mathrm{TiO}_{2} / \mathrm{SiO}_{2} / \mathrm{Si}$ substrates, subsequently pyrolized, and finally crystallized [91]. By controlling sol-gel procedure, i.e., the concentration of the stock solution, different grain size and grain size distributions, ranging from $200 \mathrm{~nm}$ to about $10 \mathrm{~nm}$ in diameter and $20 \mathrm{~nm}$ down to $2 \mathrm{~nm}$ in height were obtained. PFM measurements revealed an apparent dissappearance of the contrast indicating possible size effect in grains finer than $20 \mathrm{~nm}$. An example of the PFM measurements on such structure is shown in Figure 22.

Although self-assembly methods have the advantage of being noninvasive, one major drawback remains as the registration and size distribution of the deposited nanograins is governed by thermodynamics and, therefore, is random. Based on the fact that ultra-thin $\mathrm{TiO}_{2}$ seed layers promote the nucleation and growth of (111) textured PZT films on (111) oriented platinized substrates, Schneller et al. proposed a template technique to gain control on the arrangement of perovskite structures in the nanoscale regime prepared by CSD [90]. The idea was to prepare predefined nanosized $\mathrm{TiO}_{2}$ patterns onto a bottom electrode to act as seeds for the subsequent deposition of $\mathrm{PbTiO}_{3}$ or PZT. PFM measurements turned out to be very useful for studying size effects in self-assembled films, too.

\subsection{Imprint and Fatigue in FERAM Capacitors}

As was shown above, the functioning of several ferroelectric devices is determined by domain switching and reorientation. Therefore, the domain studies are of great practical importance. This concerns mainly ferroelectric random access memories (FeRAM) in which domain studies are indispensable for the understanding of mechanisms of polarization fatigue and imprint. Fatigue is defined as a loss of switchable polarization under prolonged cycling with a strong ac or pulsed electric 


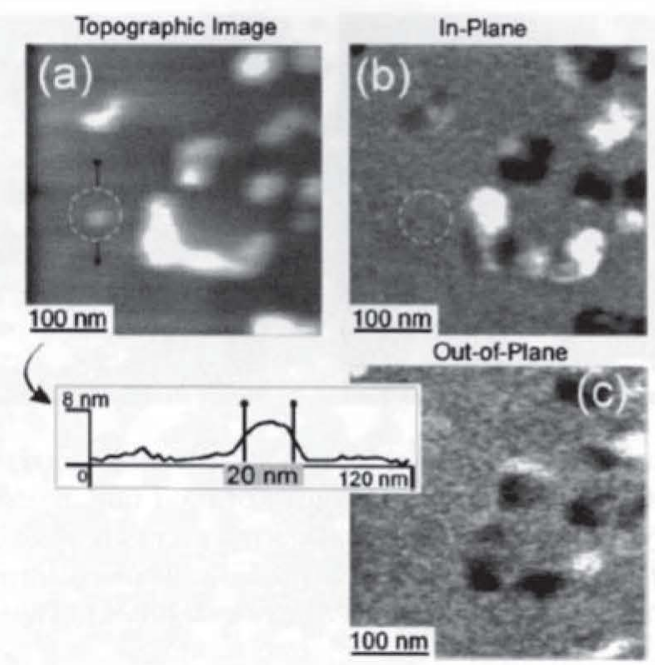

Fugure 22. The tapographical image (a) shows several PbTOO $_{3}$ grains of sizes between 100 num dowt to 20 nith, indicated by circles. In the line scan aver the grain denoted with an arrow, shown in the bottom, the size of the grain can be determined. In the PFM images (b) in-plane and (c) out-of-plane pizzoresponge of 20 nm grain is not visible. the PFM image (b) the grains of $18 \mathrm{~nm}$ (indicated by the circles) are not visible. Reprinted with permission from [91]. Copyright 2002, American Institute of Physics.

field. The problem of fatigue is difficult to solve because it involves a complex of interrelated physical and chemical phenomena occurring mainly at the interface belween the electrode and ferroelectric thin film. Until recently, the direct measurements of domain configurations before and after fatigue were impossible to do because of the lack of suitable technique for domain visualization. Only with the advent of PFM, it became feasible to address this issue. Gruverman et al. [92] first observed an effect of fatigue on the domain structure of PZT films on PT-coated substraltes, When applying a high ac voltage to the tip, continuous switching was. imitated. It has been calculated that each point received about $10^{3}$ switching cy. cles, After this, a de poling was done to the same area in an attempt to switch all ferrolectric domains. As seen in Figure 23, not all domains were switched into the "negative" (bright) state.

These observations [92] have proved that fatigue is always accompanied by strong domain wall pining, so that certain domains become frozen and do not participate in switching anymore. The results were complemented by local piesoelectric hysteresis measurement that showed a progressive shift of the $d_{33}$ loop in both vertical and horizontal directions (observed also by macroscopic technique [67]). This shift corresponds to a significant imprint of the polarization state one polarization state is preferred over the other).

Later, Colla et al. [93] confirmed the results by Gruverman and co-authors [92] using a removable electrode. In this way, the problem of inhomogeneous electric 
(a)

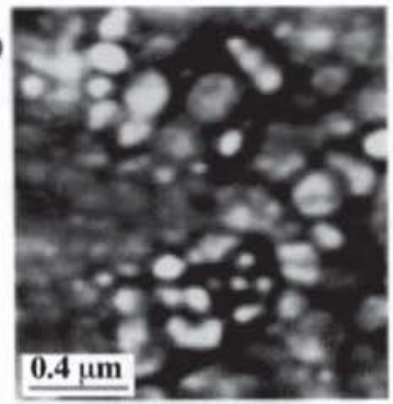

(b)

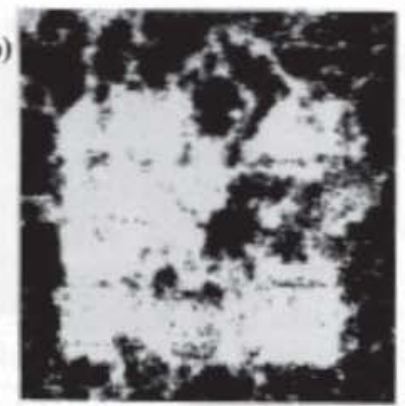

FIGURE 23. Simultaneously obtained (a) topographic and (b) piezoresponse images of the fatigued PZT/Pt heterostructure. The bright square of $1 \times 1 \mu \mathrm{m}^{2}$, produced by moving the PFM tip while applying a negative $15 \mathrm{~V}$ dc voltage, represents a negatively polarized region. Unswitchable crystallites appear as dark areas inside the white square region. Reproduced with permission from [92]. Copyright 1996, American Institute of Physics.

field was alleviated since PZT films were fatigued in real (FERAM) functioning conditions. The image was taken in "normal" PFM conditions (see Figure 3(a)) with the resolution limited only by the tip diameter.

\subsection{Ferroelectric Relaxors by PFM}

Recently, ferroelectric relaxor materials such as $\mathrm{PbMg}_{1 / 3} \mathrm{Nb}_{2 / 3} \mathrm{O}_{3}(\mathrm{PMN}), \mathrm{PbZn}_{1 / 3}$ $\mathrm{Nb}_{2 / 3} \mathrm{O}_{3}(\mathrm{PZN})$ and its solid solutions with $\mathrm{PbTiO}_{3}$ became objects of increasing interest due to their high dielectric and electromechanical properties. Exceptionally high electrostrictive and piezoelectric strain of relaxor materials is especially attractive because of the absence of hysteresis, which is detrimental to high-precision devices. It is widely accepted that unusual piezoelectric, ferroelectric, and dielectric properties of relaxors are determined by the dynamics of nanoscale polar clusters that appear far above the temperature of the dielectric maximum. These clusters may coarsen and coalesce at low temperatures, but normal ferroelectric domains may develop only after poling with a dc bias. PFM is ideally suited for studying relaxors since the effective tip diameter can be as small as the polarization cluster size and the polarization value can be assessed through $\mathrm{d}_{33}$. An example of the PFM study in PMN-0.2PT single crystals [94] is given in Figure 24.

The coexistence of rhombohedral domains (elongated strips) with remnants of polar clusters (small nanodomains embedded in between) is evident in this picture. It also illustrates the fractal nature of micron-sized domains when disorder is present. Nanodomains were shown to be important for the understanding of giant piezoelectric response in PZN-based materials [95]. In this work, nanodomains were only observed in PZN-0.045PT single crystals of certain crystallographic orientations, where the giant $d_{33}$ is found. 


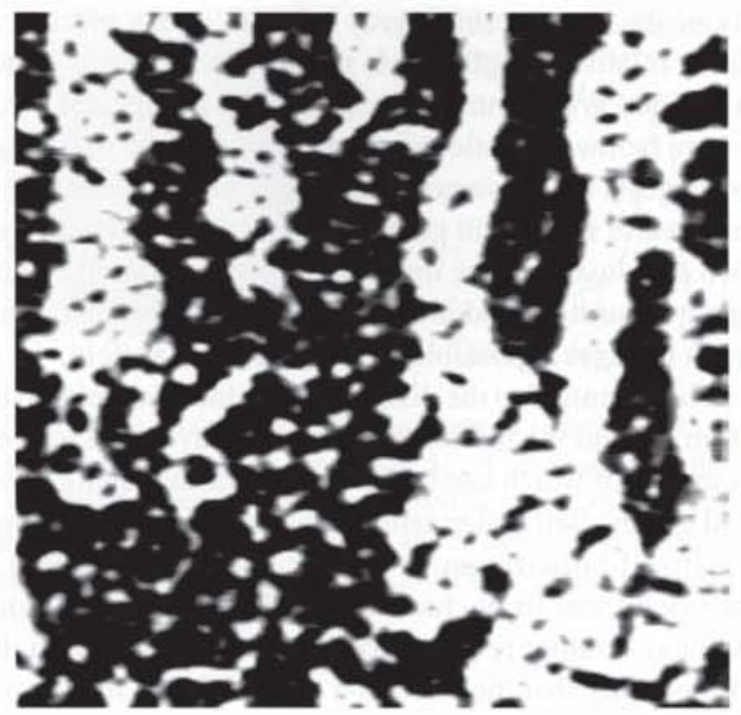

FIGURE 24. Domain structure in PMN-20\%PT single crystal illustrating a coexistence of "normal" micron-sized domains with remnants of polar clusters appearing due to random fields induced by B-site nonstoichiometry. The scan size is $5 \times 5 \mu \mathrm{m}^{2}$. Reprinted with permission from [94]. Copyright 2004, American Physical Society.

In relaxor films the switching into the terroelectric state was found to be influenced by the properties of the individual grains (due to their different orientation, chemical composition, mechanical stress, etc.). Typical topography and piezoelectric images of PMN-0.1PT films are presented in Figure 25 [96].

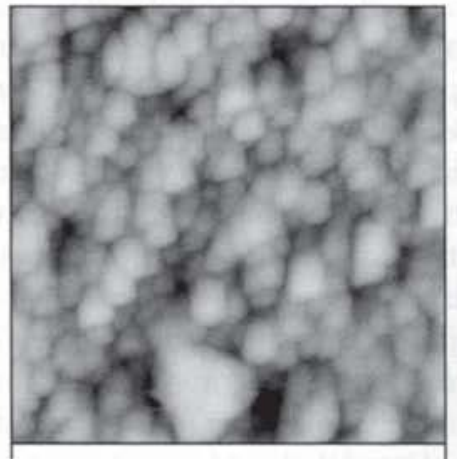

0

5.00 нм

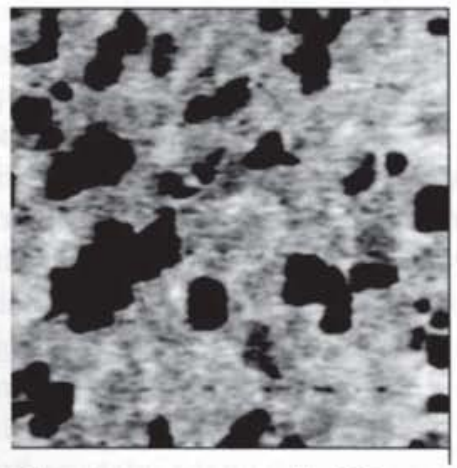

$5.00 \mathrm{\mu m}$

FIGURE 25. Topography (left) and piezoresponse image (right) of PMN-0.1PT film prepared by laser ablation. Reproduced with permission from [96]. Copyright 2002, American Institute of Physics. 
The majority of the grains exhibit gray contrast (weak piezoelectric activity), while others have relatively high $d_{33}$. It means that they are strongly polarized without any bias. Only gray contrast is expected for the relaxor ferroelectric even for the temperature below the dielectric maximum. However, strong dark contrast in some grains suggests the existence of highly polarized material.

Those grains possess significant polarization without external bias, i.e. are selfpolarizated. Two conclusions were made: (i) self-polarization can be found at the nanoscale, while it is hardly found in microscopic measurements; and (ii) since the piezoelectricity in relaxors is possible only under the bias field, the distribution of self-polarization represents also the distribution of internal field in these materials. Thus, relaxor thin film on the microscopic level behaves as a nanocomposite, the macroscopic response of which can be derived by the averaging of local responses of the individual grains. Self-polarization was recently found in pure PMN films [97], proving that self-polarization in relaxor films is not related to local composition inhomogneity due to fluctuations of $\mathrm{PbTiO}_{3}$ content. Domain structure in single-crystal and ceramic relaxors has been consequently studied by several groups [98-100] proving that the PFM is a powerful tool for the investigation of random polarization contrast due to disorder.

\subsection{Study of Piezoelectric Semiconductors by PFM}

The piezoelectric character of III-nitride semiconductors yields a novel degree of freedom in designing devices for modern micro- and nanoelectronic applications [101]. As such, measurement of the piezoelectric properties in these materials with high spatial resolution is of primary importance for the design and performance of GaN-based electronic and optoelectronic devices. It follows that the investigation of the piezoelectric behavior of nitride thin films and heterostructures at the nanoscale level is a prerequisite for determining how interfaces, dislocations, stacking faults, and inversion domain boundaries can affect electrical properties. It is known that in the wurzite phase GaN and other similar materials have sufficiently high piezoelectric response depending on whether $\mathrm{Ga}$ or $\mathrm{N}$ terminates the surface and on the quality of the material. The local piezoelectric coefficients can be thus serve as a figure of merit for various applications. The lower values of piezoelectric coefficients ( $d_{33}$ is much smaller than in PZT) can be counteracted by applying relatively high ac-voltage to the tip. No switching is expected in piezoelectric semiconductors since they are not ferroelectric.

The example of the polarization imaging is shown in Figure 26 for GaN thin films deposited either directly on sapphire substrate ( $\mathrm{N}$ termination) or on the AIN nucleation layer (Ga face) [102]. The film was deposited in such a way that both terminations are seen on the image as bright and dark areas.

By imaging GaN with PFM it was possible to judge the quality of the interface by measuring piezoelectric coefficients, since they are influenced by stress. It was shown that the quality of the Ga-terminated surface is lower than that for $\mathrm{N}$-face. 


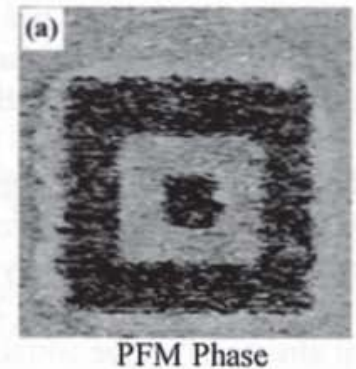

(b) PFM Phase Histogram

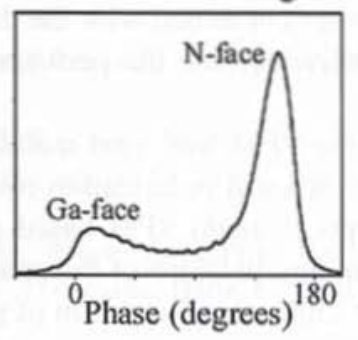

FIGURE 26. (a) PFM phase image of a GaN-based lateral polarity heterostructure, (d) histogram of the PFM phase image. The innermost $5 \times 5 \mu \mathrm{m}^{2}$ square is the Ga-face region. There is a sharp contrast difference in the piezoresponse phase image, demonstrating inversion in film polarity. The magnitudes of the $\mathrm{Ga}$ - and $\mathrm{N}$-face peaks correspond to the relative areas of each region. Reprinted with permission from [102]. Copyright 2002, American Institute of Physics.

\section{Outlook}

As shown above, during the past decade piezoelectric force microscopy has grown from a mere imaging tool for domain visualization in ferroelectric materials to a powerful technique for quantitative characterization and manipulation in polar materials at the nanoscale. PFM is currently being implemented in many commercial systems (see, e.g., [103]) with a possibility of adding additional modes (imaging mode, piezoelectric spectroscopy, hysteresis, etc). Recently demonstrated usefulness of PFM for imaging of biological materials such as electromechanically active proteins in calcified and connecting issues [104] suggests a much broader range of materials (other than ferroelectric) that can be investigated by this technique. Below we will briefly mention some new challenges offered by this increasingly important measurement and manipulation technique.

The crucial part of the PFM setup is the shape and material of the probe in contact with the ferroelectric surface. In order to be used as a movable electrode, the electrical and mechanical properties of the probe as well as its shape should be carefully controlled. Conventional probes (metal-coated or made from doped Si) all suffer from metal peel-off, contamination with hydrocarbonates and other species on the surface, and uncontrolled shape. Also, the resistivity of the doped Si probes is not low enough for quantitative determination of piezoelectric coefficients at the nanoscale. Platinum (frequently used as a metal) causes electrochemical reactions on the surface of ferroelectric oxides, thus resulting in deterioration of contrast and loss of resolution. We envisage that the development of all-metal probes or Si with implanted metal coatings will drastically increase the capabilities of PFM allowing, for example, the measurements at high temperatures or in different environments. The resolution (determined by the effective diameter of the probe) 
can be increased by using diamond tips with controlled shape and aspect ratio. These new probes combined with the thorough control of the surface conditions should drastically increase the performance of PFM with a potential of atomic resolution.

Until now, the PFM was used mainly as a high-resolution imaging tool, but the advantages of local polarization reversal have never been utilized except of the few attempts to build SPM-based ferroelectric data storage systems [105]. With the continuing increase of the spatial resolution, PFM can be also used for a new task: i.e., intentional creation of polar structures on the surface for assembling various nanostructures via a so-called polarization-driven mechanism [106]. It has been long known that the chemical reactivity of ferroelectric surfaces depend on the polarization direction (e.g., the dissolution in acids is always depend whether positive or negative end of domain is etched). Recently, the combination of polarization-dependent chemical reactivity and domain patterning gave rise to a novel technology called ferroelectric lithography [106]. The advantage of this lithography is that, depending on mechanism of adsorption, a variety of nanostructures can be, in principle, built. Further, local polarization patterning on the scale of few nanometers can be used for the recognition of specific polar molecules and groups of many biologically important materials, such as glycolipids or certain proteins. Along with sub-nanometer resolution for probing biological materials via piezoelectric or electrostrictive effects [104], it provides a powerful tool to modern nanotechnology.

\section{References}

1. J. F. Scott, Ferroelectric Memories (Springer, Heidelberg, 1993).

2. A. Gruverman in Encyclopedia of Nanoscience and Nanotechnology, edited by H. S. Nalwa (American Scientific Publishers, Los Angeles. 2004) Vol. 3, pp. 359-375.

3. L. M. Eng, M. Bummerlin, C. Loppacher, M. Guggisberg, R. Bennewitz, R. Luthi, E. Meyer, T. Huser, H. Heinzelmann, and H.-J. Guntherodt, Ferroelectrics 222, 153 (1999).

4. A. Gruverman, O. Auciello, and H. Tokumoto, Annu. Rev. Mater. Sci. 28, 101 (1998).

5. M. Abplanalp, L. Eng, and H. Güntherodt, Appl. Phys. A 66, S231 (1998).

6. E. L. Colla, S. Hong, D. V. Taylor, A. K. Tagantsev, N. Setter, and K. No, Appl. Phys. Lett. 72, 2763 (1998).

7. A. Roelofs, U. Boettger, R. Waser, F. Schlaphof, S. Trogisch, and L. Eng, Appl. Phys. Lett. 77, 3444 (2000).

8. S. V. Kalinin and D. A. Bonnell, Phys. Rev. B 65, 125408 (2002).

9. C. S. Ganpule, A. L. Roytburd, V. Nagarajan, B. K. Hill, S. B. Ogale, E. D. Williams, R. Ramesh, and J. F. Scott, Phys. Rev. B 65, 014101 (2002).

10. C. Loppacher, F. Schlaphof, S. Schneider, U. Zerweck, S. Grafstrom, L. M. Eng, A. Roelofs, and R. Waser, Surface Science 483, 532, (2003).

11. P. Guthner and K. Dransfeld, Appl. Phys. Lett. 61, 1137 (1992).

12. M. Alexe and A. Gruverman (eds.), Nanoscale Characterization of Ferroelectric Materials (Springer-Verlag, Berlin, 2004). 
13. S. Hong (ed.), Nanoscale Phenomena in Ferroelectric Thin Films (Academic Publishers, Norwell MA, 2004).

14. L. Eng, H. Güntherod, G. Schneider, U. Köpke, and J. M. Saldana, Appl. Phys. Lett. 74, 233 (1999).

15. C. S. Ganpule, V. Nagarajan, B. K. Hill, A. L. Roytburd, E. D. Williams, R. Ramesh, S. P. Alpay, A. Roelofs, R. Waser, and L. M. Eng, J. Appl. Phys. 91, 1477 (2002).

16. C. Durkan, M. Welland, D. Chu, and P. Migliorato, Appl. Phys. Lett. 76, 366 (2000).

17. C. Harnagea, Ph.D. thesis, Martin-Lüther-Universität Halle, Germany, 2001.

18. S. V. Kalinin, Ph.D. thesis, University of Pennsylvania, USA, 2002.

19. S. Hong, J. Woo, H. Shin, J. U. Jeon, Y. E. Pak, E. Colla, N. Setter, E. Kim, and K. No, J. Appl. Phys. 89, 1377 (1998).

20. M. Abplanalp, Ph.D. thesis, Swiss Federal Institute of Technology, Switzerland, 2001.

21. E. Luo, Z. Xie, J. Xu, I. Wilson, and L. Zhao, Phys. Rev. B 61, 203 (2000).

22. J. W. Hong, K. H. Noh, S. Park, S. I. Kwun, and Z. G. Khim, Phys. Rev. B 58, 5078 (1998).

23. S. V. Kalinin and D. A. Bonnell, Appl. Phys. Lett. 78, 1116 (2001).

24. F. Peter, K. Szot, R. Waser, B. Reichenberg, S. Tiedke, and J. Szade, Appl. Phys. Lett. 85, 2896 (2004).

25. M. Abplanalp, J. Fousek, and P. Günter, Phys. Rev. Lett. 86, 5799 (2001).

26. G. Zavala, J. H. Fendler, and S. McKinstry, J. Appl. Phys. 81, 7480 (1997).

27. A. L. Kholkin, V. V. Shvartsman, A. Y. Emelyanov, R. Poyato, M. L. Calzada, and L. Pardo, Appl. Phys. Lett. 82, 2127 (2003).

28. A. Y. Emelyanov, N. A. Pertsev, and A. L. Kholkin, Phys. Rev. B 66, 214108 (2002).

29. K. Prume, A. Roelofs, T. Schmitz, B. Reichenberg, S. Tiedke, and R. Waser, Jpn. J. Appl. Phys. 41, 7198 (2002).

30. J. W. Hong, D. S. Kahng, J. C. Shin, H. J. Kim, and Z. G. Khim, J. Vac. Sci. Technol. B 16, 2942 (1998).

31. U. Rabe, K. Janser, and W. Amold, Rev. Sci. Instrum. 67, 3281 (1996).

32. B. Jaffe, W. R. Cook, and H. Jaffe, Piezoelectric Ceramics (Academic, London, 1971).

33. C. Harnagea, A. Pignolet, M. Alexe, D. Hesse, Integr. Ferroelectr. 38, 667 (2001).

34. S. V. Kalinin, B. J. Rodriguez, S. Jesse, J. Shin, A. P. Baddorf, P. Gupta, H. Jain, D. B. Williams, and A. Gruverman. Microscopy and Microanalysis 12, 206 (2006).

35. T. Tybell, C. H. Ahn, and J.-M. Triscone, Appl. Phys. Lett. 75, 856 (1999).

36. T. Tybell, P. Paruch, T. Giamarchi, and J.-M. Triscone, Phys. Rev. Lett. 89, 097601 (2002).

37. K. Terabe, M. Nakamura, S. Takekawa, K. Kitamura, S. Higuchi, Y. Gotoh, and Y. Cho, Appl. Phys. Lett. 82, 433 (2003).

38. H. F. Hamann, Y. C. Martin, and H. K. Wickramasinghe, Appl. Phys. Lett. 84, 810 (1994).

39. Y. Cho, S. Hashimoto, N. Odagawa, K. Tanaka, and Y. Hiranaga, Appl. Phys. Lett. 87, 232907 (2005).

40. Y. Rosenwaks, D. Dahan, M. Molotskii, and G. Rosenman, Appl. Phys. Lett. 86, 012909 (2005).

41. F. Jona and G. Shirane, Ferroelectric Crystals (Dover Publications, New York, 1993).

42. J. Giocondi and G. S. Rohrer, Chem. Mater. 13, 241 (2001).

43. S. V. Kalinin, D. A. Bonnell, T. Alvarez, X. Lei, Z. Hu, J. H. Ferris, Q. Zhang, and S. Dunn, Nano Letters 2, 589 (2002). 
44. S. V. Kalinin, D. A. Bonnell, T. Alvarez, X. Lei, Z. Hu, R. Shao, and J. H. Ferris, Adv. Mat. 16, 795 (2004).

45. X. Liu, K. Terabe, M. Nakamura, S. Takekawa, and K. Kitamura, J. Appl. Phys. 97, $064308(2005)$.

46. M. Molotskii, A. Agronin, P. Urenski, M. Shvebelman, G. Rosenman, and Y. Rosenwaks, Phys. Rev. Lett. 90, 107601 (2003).

47. M. Molotskii, J. Appl. Phys. 93, 6234 (2003).

48. S. V. Kalinin, E. Karapetian, and M. Kachanov, Phys. Rev. B 70, 184101 (2004).

49. S. V. Kalinin, A. Gruverman, B. J. Rodriguez, J. Shin, A. P. Baddorf, E. Karapetian, and M. Kachanov, J. Appl. Phys. 97, 074305 (2005).

50. A. N. Morozovska and E. A. Eliseev, Phys. Stat. Sol. (b) 242, R79 (2005).

51. A. Y. Emelyanov, Phys. Rev. B 71, 132102 (2005).

52. M. I. Molotskii and M. M. Shvebelman, Philos. Magaz. A 85, 1637 (2005).

53. A. N. Morozovska and E. A. Eliseev, Physica B 373, 54 (2006).

54. Eq. (3) is valid only for $l_{d}>r_{d}$. To avoid this limitation, used here was the expression for the demagnetization factor for prolate ellipsoid from J. A. Osborn, Phys. Rev. 67, 351 (1945).

55. R. Landauer, J. Appl. Phys. 28, 227 (1957).

56. J. Li, B. Nagaraj, H. Liang, W. Cao, C. H. Lee, and R. Ramesh, Appl. Phys. Lett. 84, 1174 (2004).

57. C. Dehoff, B. J. Rodriguez, A. I. Kingon, R. J. Nemanich, A. Gruverman, and J. S. Cross, Rev. Sci. Instrum. 76. 023708 (2005).

58. P. Paruch, T. Giamarchi, and J.-M. Triscone, Phys. Rev. Lett. 94, 197601 (2005).

59. B. J. Rodriguez, R. J. Nemanich, A. Kingon, A. Gruverman, S. V. Kalinin, K. Terabe, X. Y. Liu, and K. Kitamura, Appl. Phys. Lett. 86, 012906 (2005).

60. A. Gruverman, B. J. Rodriguez, C. Dehoff, J. D. Waldrep, A. I. Kingon, R. J. Nemanich, and J. S. Cross, Appl. Phys. Lett. 87, 082902 (2005).

61. A. N. Kolmogorov, Izv. Akad. Nauk SSSR, Ser. Math. 3, 355 (1937).

62. W. Jo, D. C. Kim, and J. W. Hong, Appl. Phys. Lett. 76, 390 (2000).

63. A. Gruverman, H. Tokumoto, A. S. Prakash, S. Aggarwal, B. Yang, M. Wuttig, R. Ramesh, O. Auciello, and T. Venkatesan, Appl. Phys. Lett. 71, 3492 (1997).

64. A. Gruverman and M. Tanaka, J. Appl. Phys. 89, 1836 (2001).

65. C. S. Ganpule, A. L. Roytburd, V. Nagarajan, B. K. Hill, S. B. Ogale, E. D. Williams, R. Ramesh, and J. F. Scott, Phys. Rev. B 65, 014101 (2001).

66. A. L. Kholkin, E. K. Akdogan, A. Safari, P.-F. Chauvy, and N. Setter, J. Appl. Phys. 89, 8066 (2001).

67. A. L. Kholkin, E. L. Colla, A. K. Tagantsev, D. V. Taylor, and N. Setter, Appl. Phys. Lett. 68, 2577 (1996).

68. Y. Zhang, I. S. Baturin, E. Aulbach, D. C. Lupascu, A. L. Kholkin, V. Y. Shur, and J. Rödel, Appl. Phys. Lett. 86, 012910 (2005).

69. V. V. Shvartsman, N. A. Pertsev, J. M. Herrero, C. Zaldo, and A. L. Kholkin, J. Appl. Phys. 97, 104105 (2005).

70. S. V. Kalinin, A. Gruverman, and D. A. Bonnell, Appl. Phys. Lett. 85, 795 (2004).

71. A. L. Kholkin, I. K. Bdikin, V. V. Shvartsman, A. Orlova, D. Kiselev, and V. Bogomolov, MRS Proceedings 838E, O7.6 (2005).

72. A. Gruverman, A. Pignolet, K. M. Satyalakshmi, M. Alexe, N. D. Zakharov, and D. Hesse, Appl. Phys. Lett. 76, 106 (2000).

73. A. Wu, P. M. Vilarinho, G. Suchaneck, and A. L. Kholkin, Nanotechnology 16, 2587 (2005). 
74. S. V. Kalinin, Appl. Phys. Lett. (submitted).

75. S. V. Kalinin and D. A. Bonnell, Phys. Rev. B 63, 125411 (2001).

76. C. S. Ganpule, V. Nagarjan, H. Li, A. S. Ogale, D. E. Steinhauer, S. Aggarwal, E. Williams, R. Ramesh, and P. Wolf, Appl. Phys. Lett. 77, 292 (2000).

77. P. Gupta, H. Jain, D. B. Williams, S. V. Kalinin, J. Shin, and A. P. Baddorf, Appl. Phys. Lett. 87, 172903 (2005).

78. R. Waser (ed), Nanoelectronics and Information Technology: Advanced Electronic Materials and Novel Devices (Wiley-VCH, Berlin, 2005).

79. J. F. Scott, H. M. Duiker, P. D. Beale, B. Pouligny, K. Dimmler, M. Parris. D. Butler, and S. Eaton, Physica B+C 150, 160 (1988).

80. P. Ghosez and K. M. Rabe, Appl. Phys. Lett. 76, 2767 (2000).

81. A. Stanishevsky, S. Aggarwal, A. Prakash, J. Melngailis, and R. Ramesh, J. Vac. Sci. Technol. B 16, 3899 (1998).

82. C. Ganpule, A. Stanishevsky, S. Aggarwal, J. Melngailis, E. Williams, R. Ramesh, V. Joshi, and C. P. Araujo, Appl. Phys. Lett. 75, 3874, (1999).

83. C. Ganpule, A. Stanishevsky, Q. Su, S. Aggarwal, J. Melngailis, E. Williams, and R. Ramesh, Appl. Phys. Lett. 75, 409 (1999).

84. S. Bühlmann, B. Dwir, J. Baborowski, and P. Muralt, Appl. Phys. Lett. 80, 3195 (2002).

85. H. Craighead and L. Schiavone, Appl. Phys. Lett. 48, 1748 (1989).

86. M. Alexe, C. Harnagea, W. Erfurth, D. Hesse, and U. Gösele, Appl. Phys. A 70, 247 (2000).

87. A. Seifert, A. Vojta, J. S. Speck, and F. F. Lange, J. Mat. Res. 11, 1470 (1996).

88. H. Fujisawa, K. Morimoto, M. Shimizu, and H. Niu, Jpn. J. Appl. Phys. 39, 5446 (2000).

89. R. Waser, T. Schneller, S. Hoffmann-Eifert, and P. Ehrhart, Integr. Ferroelectr. 36, 3 (2001).

90. T. Schneller and R. Waser, Ferroelectrics 267, 293 (2002).

91. A. Roelofs, T. Schneller, K. Szot, and R. Waser, Appl. Phys. Lett. 81, 5231 (2002).

92. A. Gruverman, O. Auciello, and H. Tokumoto, Appl. Phys. Lett. 69, 3191 (1996).

93. E. L. Colla, I. Stolichnov, P. E. Bradely, and N. Setter, Appl. Phys. Lett. 82, 1604 (2003).

94. V. V. Shvartsman and A. L. Kholkin, Phys. Rev. B 69, 014102 (2004).

95. I. K. Bdikin, V. V. Shvartsman, and A. L. Kholkin, Appl. Phys. Lett. 83, 4232 (2003).

96. V. V. Shvartsman, A. Y. Emelyanov, A. L. Kholkin, and A. Safari, Appl. Phys. Lett. 81, 117 (2002).

97. V. V. Shvartsman, A. L. Kholkin, M. Tyunina, and J. Levoska, Appl. Phys. Lett. 86, 222907 (2005).

98. S. B. Vakhrushev, A. A. Naberezhnov, B. Dkhil, J.-M. Kiat, V. Shwartsman, A. Kholkin, B. Dorner, and A. Ivanov, AIP Conf. Proc. 677, 74 (2003).

99. F. Bai, J. Li, and D. Viehland, Appl. Phys. Lett. 85, 2313 (2004).

100. V. V. Shvartsman, M. Wojtas, S. Vakhrushev, and A. L. Kholkin, Mat. Res. Soc. Symp. Proc. 785, D4.11 (2004).

101. O. Ambacher, J. Majewski, C. Miskys, A. Link, M. Hermann, M. Eickhoff, M. Stutzmann, F. Bernardini, V. Fiorentini, V. Tilak, B. Schaff, and L. F. Eastman, J. Phys.: Condens. Matt. 14, 3399 (2002).

102. B. J. Rodriguez, A. Gruverman, A. I. Kingon, and R. J. Nemanich, Appl. Phys. Lett. 80, 4166 (2002). 
103. http://www.ntmdt.ru/SPM-Techniques

104. B. J. Rodrigues, S. V. Kalinin, J. Shin, J. Jesse, V. Grichko, T. Thundat, A. P. Baddorf, and A. Gruverman, J. Appl. Phys. (in press).

105. Y. Hiranaga and Y. Cho, Jpn. J. Appl. Phys. 44, 6960 (2005).

106. S. V. Kalinin, D. A. Bonnell, T. Alvarez, X. Lei, Z. Hu, R. Shao, and J. H. Ferris, Adv. Mat. 16, 795 (2004). 